\title{
Quantification and Optimization of Dynamic Kinetic Resolution
}

\author{
John Andraos* \\ Department of Chemistry, York University, Toronto, ON M3J 1P3, Canada \\ Received: October 17, 2002
}

\begin{abstract}
A complete and exact kinetic analysis of the phenomenon of dynamic kinetic resolution is presented. This analysis is applicable to reactions of stable stereoisomeric substrates whose ratios can be controlled and is valid for any set of kinetic conditions within the constraint of first-order and pseudo-first-order processes. Two new linear relationships are found for the dependence of the initial product ratio on the initial substrate ratio and for the dependence of the final product excess on the initial substrate excess. These relationships yield the minimum number of rate constant ratios needed to characterize the energetics of a chemical system exhibiting dynamic kinetic resolution completely. A distinct experimental advantage of this method is that it is based entirely on product studies. A simple graphical representation of the second linear relationship depicts visually the limiting Curtin-Hammett and anti-Curtin-Hammett conditions. From these conditions, a new parameter is defined that characterizes the efficiency of dynamic kinetic resolution and Curtin-Hammett efficiency. Simulations based on enantiomeric substrates illustrate how reactions may be optimized using this graphical treatment. An extension of this analysis to related kinetic schemes of varying degrees of complexity shows that the above linear relationships are universal. Results from these treatments are compared with Noyori's quantitative work on the stereoselective hydrogenation of $\beta$-ketoesters. Implications of this new analysis are also discussed in light of previous work done on the applicability of the Winstein-Holness and Curtin-Hammett approximations to reactions of substrates that are interconverting conformers. For these cases, an alternate definition of Curtin-Hammett efficiency is proposed that is based on the experimental determination of the initial and final product ratios and the equilibrium constant for substrate interconversion. This unified analysis can be readily applied to a wide variety of synthetic and mechanistic problems in organic chemistry where dynamic kinetic resolution is applicable.
\end{abstract}

\section{Introduction and Background}

The resolution of chiral substrates and products is of fundamental importance in the stereoselective synthesis of optically pure materials, particularly pharmaceuticals. The connection between the optical purity and function of a drug in biological systems was made most dramatically by the thalidomide tragedy in the early 1960 s. ${ }^{1}$ Kinetic,${ }^{2}$ dynamic kinetic, ${ }^{3}$ and more recently dynamic thermodynamic ${ }^{4}$ resolutions have all been used as strategies to effect the syntheses of optically pure molecules. Another chemical phenomenon of fundamental interest is the synthesis of different products from a common substrate that exists in different conformations, where each product arises from a specific conformer. The key kinetic model, shown in Scheme 1, describing both of these situations is the one on which the Curtin-Hammett principle was postulated nearly 50 years ago. ${ }^{5 a}$ Substrates $\mathrm{X}$ and $\mathrm{Y}$ may be either different conformers of a single substance or a pair of racemizable enantiomers or diasereomers. Though the original paper by Curtin contains no mathematical expressions relating product ratios to rate constants, several workers have made attempts to quantify this principle since 1954.

Most notably, Zefirov 6 and Seeman and Farone $^{7}$ have provided analytical solutions to Scheme 1 under first-order and pseudo-first-order conditions. Zefirov derived an expression for the final product ratio when the substrates are initially at equilibrium. Such a solution is applicable to the case of substrates that are interconverting conformers. Seeman and

\footnotetext{
*E-mail: jandraos@yorku.ca.
}

\section{SCHEME 1}

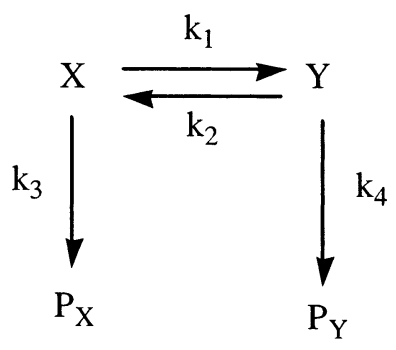

Farone presented an exact solution for the concentration-time dependencies of all four species and discussed the range of validity of the Winstein-Holness and Curtin-Hammett approximations on the basis of this solution. They were able to conclude that both approximations were valid so long as $k_{1}$ and $k_{2}$ were both at least an order of magnitude larger than $k_{3}$ and $k_{4}$. This finding is consistent with the majority of documented reactions obeying Scheme 1 involving the slow decomposition of fast interconverting substrates. The analytical solution to Scheme 1 is a special case of the more complicated one given in two reports 8 preceding those of Zefirov and Seeman involving all possible reversible reactions between four chemical species.

The central difficulty with these exact analytical treatments, however, is that they have not been widely utilized by synthetic organic chemists in their efforts to optimize syntheses of important stereochemical compounds or by mechanistic chemists in the determination of relative energy barriers for chemical systems obeying Scheme 1. Even when exact solutions become 
available, Winstein-Holness and Curtin-Hammett conditions are instead invoked to justify experimental results since they naturally lead to simpler accessible expressions. The shortcomings of these approximations in obtaining quantitative data have been well documented. It is not surprising then that it has been challenging to quantify relative energy barriers in CurtinHammett-type schemes experimentally, particularly when they are applied to synthetic problems involving dynamic kinetic resolution. This situation has changed little since Seeman's extensive historical review ${ }^{7 \mathrm{~b}}$ of Curtin-Hammett /WinsteinHolness kinetics in which Zefirov's early comments to break the impasse are noted. Therefore, the reinvestigation of the analytical treatment of the fundamental Curtin-Hammett scheme and its extended variants is warranted so as to bring about a workable marriage between theory and practice. In particular, the main goal here is to obtain useful parameters from mathematical treatments that can be readily accessed from experimental data.

The early conformational studies by Barton, Cram, Winstein, and others centered on the problem of correlating the populations of products with the populations of their precursor substrates. The experimental paradigm followed for characterizing the energetics of such chemical systems centered on first obtaining equilibrium constants for substrate inversions and/or racemizations in the absence of any other reactants. Then, in separate kinetic experiments, the reactivity of the starting equilibrated substrate mixture was investigated. Specifically, final product ratios and the global rate constant for the appearance of all products originating from both substrates were determined. Both the Winstein-Holness equation and the Curtin-Hammett approximation were then used to determine the key rate constant ratio, $k_{3} / k_{4}$. The justification for using both of these approximations was usually made by performing independent molecular mechanics or other theoretical calculations to estimate energy barriers for product forming and substrate equilibration steps. The net result is that not all of the evidence is provided entirely by experiment. The missing link in these approaches is that experimental determination of any one of the "cross" ratios $k_{1} /$ $k_{3}, k_{1} / k_{4}, k_{2} / k_{3}$, or $k_{2} / k_{4}$ needed to validate the approximations was not made.

The connection between traditional conformational studies and the present interest in kinetic and dynamic kinetic resolution has recently been vigorously investigated. ${ }^{3}$ Most notably, Kagan, ${ }^{9}$ Noyori, ${ }^{10}$ Blackmond, ${ }^{11}$ and Singleton ${ }^{12}$ have independently investigated nonlinear effects in kinetic resolutions using enantioimpure catalysts. It is important to note that these treatments describe kinetic models that differ fundamentally from those of Scheme 1. The reactions studied by these workers involve achiral substrates reacting with enantioimpure mixtures of nonracemizable chiral catalysts. An example reaction first analyzed by Noyori is the asymmetric alkylation of aromatic aldehydes with dialkylzincs using amino-alcohol catalysts to achieve chiral amplification. Unlike Scheme 1, there is no dynamic component in the substrate, and no racemization of any one catalyst of a given stereochemistry to another occurs. Chiral products arise directly from the reaction of the substrate with any one of the chiral catalysts present in the reaction mixture that exist in monomeric and dimeric forms.

Kinetic models involving stereochemical substrates discussed in this paper describe reactions of racemizable chiral substrates with achiral catalysts (reagents) or with enantiopure chiral catalysts (reagents) under pseudo first-order conditions. The most notable work relevant to the present investigation is that of Noyori, who introduced a mathematical description of dynamic kinetic resolution on the basis of Scheme 1 by simulating the variation of enantioselectivity with percent reaction conversion under Curtin-Hammett conditions. ${ }^{13 a, b}$ Theoretically determined template curves were first generated from a series of rate constant ratios, and these were then used to estimate enantioselectivities at 0 and $100 \%$ reaction completion by overlaying them on experimental data recorded at various reaction times. Rate constant ratios were adjusted accordingly until a good visual fit was obtained. It was demonstrated that for slow enough reactions time-dependent enantioselectivities could be determined by periodically sampling reaction mixtures and analyzing their product distribution by chromatographic methods. In this way, it was possible to verify that the CurtinHammett condition was valid for the hydrogenation of $\beta$-ketoesters in the presence of BINAP-Ru(II) catalysts. Other workers ${ }^{14}$ also used similar strategies to quantify enantioselectivity. To date, however, reported experimental plots based on percent conversion suffer from too few data points to be accurately fitted to the complicated sigmoidlike Noyori functions. Moreover, these curves do not always approach minima or maxima at 0 or $100 \%$ reaction completion depending on the magnitudes of the rate constant ratios. A key feature of Noyori's analysis is that estimates of these ratios were determined from product studies based on reactions carried out at a single initial substrate condition, commonly, racemic mixtures. It will be shown in this investigation that direct and reliable estimates of rate constant ratios can be made if product study data are collected from a set of initial conditions covering the full range of possible optical purities of starting materials.

This work first presents a complete and accessible analytical treatment of Scheme 1 and related schemes using the Laplace transform or operator method. ${ }^{15}$ Pharmacokineticists in the study of drug metabolism in mammalian bodies have extensively used the Laplace transform method, largely unknown among practicing organic chemists. A detailed description of this method has recently appeared for the determination of analytical solutions to well-known unimolecular kinetic schemes. ${ }^{15 \mathrm{c}}$ What is presented in this work is an a priori determination of key rate constant ratios without invoking any approximations on the analytical solution. Simple expressions for product ratios and product excesses are determined both at initial and final reaction times. Simulations show that enantioselectivities at 0 and $100 \%$ reaction are more reliably determined by extrapolating plots of product excess versus time rather than versus percent reaction completion. Linear relationships for the dependence of the initial product ratio on the initial substrate ratio and for the dependence of the final product excess on the initial substrate excess suggest new manipulations of product study data. A graphical representation of these relationships allows for the first time the experimental determination of all rate constant ratios and the extent of dynamic kinetic resolution under any set of conditions; that is, the analysis is applicable whether the Curtin-Hammett principle holds for a given chemical system. The graphical treatment is universal and is applicable to any complex kinetic scheme that has at its core the elements of Scheme 1. A graphical simulation study also shows how a given reaction can be optimized once its relative energy barriers are fully characterized. These results also demonstrate the precise mathematical conditions of the Curtin-Hammett principle.

\section{Kinetic Analyses of Fundamental and Extended Schemes}

The time-dependent concentration profiles for substrates and products in Scheme 1 under the initial conditions $[\mathrm{X}]_{0}=a$ and $[\mathrm{Y}]_{0}=b$ are given by eqs $1 \mathrm{a}-\mathrm{d}$ 


$$
\begin{aligned}
& {[\mathrm{X}]=\left\{\frac{a\left(k_{2}+k_{4}-\gamma_{1}\right)+b k_{2}}{\gamma_{2}-\gamma_{1}}\right\} \mathrm{e}^{-\gamma_{1} t}-} \\
& \left\{\frac{a\left(k_{2}+k_{4}-\gamma_{2}\right)+b k_{2}}{\gamma_{2}-\gamma_{1}}\right\} \mathrm{e}^{-\gamma_{2} t} \\
& {[\mathrm{Y}]=\left\{\frac{b\left(k_{1}+k_{3}-\gamma_{1}\right)+a k_{1}}{\gamma_{2}-\gamma_{1}}\right\} \mathrm{e}^{-\gamma_{1} t}-} \\
& \left\{\frac{b\left(k_{1}+k_{3}-\gamma_{2}\right)+a k_{1}}{\gamma_{2}-\gamma_{1}}\right\} \mathrm{e}^{-\gamma_{2} t} \\
& {\left[\mathrm{P}_{\mathrm{X}}\right]=\frac{b k_{2} k_{3}+a k_{3}\left(k_{2}+k_{4}\right)}{\gamma_{1} \gamma_{2}}-} \\
& \left\{\frac{b k_{2} k_{3}+a k_{3}\left(k_{2}+k_{4}-\gamma_{1}\right)}{\gamma_{1}\left(\gamma_{2}-\gamma_{1}\right)}\right\} \mathrm{e}^{-\gamma_{1} t}+ \\
& \left\{\frac{b k_{2} k_{3}+a k_{3}\left(k_{2}+k_{4}-\gamma_{2}\right)}{\gamma_{2}\left(\gamma_{2}-\gamma_{1}\right)}\right\} \mathrm{e}^{-\gamma_{2} t} \\
& {\left[\mathrm{P}_{\mathrm{Y}}\right]=\frac{a k_{1} k_{4}+b k_{4}\left(k_{1}+k_{3}\right)}{\gamma_{1} \gamma_{2}}-} \\
& \left\{\frac{a k_{1} k_{4}+b k_{4}\left(k_{1}+k_{3}-\gamma_{1}\right)}{\gamma_{1}\left(\gamma_{2}-\gamma_{1}\right)}\right\} \mathrm{e}^{-\gamma_{1} t}+ \\
& \left\{\frac{a k_{1} k_{4}+b k_{4}\left(k_{1}+k_{3}-\gamma_{2}\right)}{\gamma_{2}\left(\gamma_{2}-\gamma_{1}\right)}\right\} \mathrm{e}^{-\gamma_{2} t}(1 \mathrm{a}-\mathrm{d})
\end{aligned}
$$

where $\gamma_{1}+\gamma_{2}=k_{1}+k_{2}+k_{3}+k_{4}$ and $\gamma_{1} \gamma_{2}=k_{1} k_{4}+k_{2} k_{3}+$ $k_{3} k_{4}$. This solution is strictly valid under first-order or pseudofirst-order conditions. A complete solution using the Laplace transform method is given in the Supporting Information. The symmetry of the forms of eqs $1 \mathrm{a}$ and $\mathrm{b}$ and of the forms of eqs $1 \mathrm{c}$ and $\mathrm{d}$ is evident. This property occurs in the forms of the inverse Laplace transforms from which the time-dependent functions are derived. The expression for [Y] can be immediately written down once that of $[\mathrm{X}]$ is known by making the following variable and subscript interchanges in each term: $a$ replaces $b$, 1 replaces 2 , and 3 replaces 4 . Precisely the same observation is made when comparing expressions for $\left[\mathrm{P}_{\mathrm{X}}\right]$ and $\left[\mathrm{P}_{\mathrm{Y}}\right]$. This kind of symmetry between pairs of concentration variables is an underlying pattern that holds for any kinetic scheme that has symmetric elementary steps. Hence, the task of obtaining expressions for the concentration-time profiles is facilitated since only half of them need to be worked out by the Laplace transform method. This technique is exploited in the determination of analytical solutions of more complex extended kinetic schemes based on Scheme 1 (see Schemes 3-9 and 11-13 in Table 1). The Supporting Information presents the sets of inverse Laplace transforms for all extended schemes discussed in this work. Since Scheme 10 is asymmetric in the pattern of elementary steps connecting the four chemical species, the time -dependent functions need to be determined separately. For the purposes of obtaining initial and final product ratios and product excesses, it is unnecessary to evaluate coefficients $\gamma_{1}$ and $\gamma_{2}$ further since these appear in the denominators of terms and cancel out when ratios are evaluated. Final product ratios are easily determined since all exponential terms drop out, leaving behind only constant terms whereas initial product ratios are determined using L'Hôpital's rule ${ }^{16}$ since evaluation by direct substitution leads to indeterminate forms (see Table S2). The advantages of casting analytical solutions in the present forms may be appreciated when they are compared with previous expressions. ${ }^{6,7}$ Furthermore, it can be shown that the WinsteinHolness equation ${ }^{17}$ is obtainable from eqs $1 \mathrm{a}-\mathrm{d}$ when $\gamma_{1}=\gamma_{2}$. (See Appendix.)

\section{Chemical Relevance of Kinetic Schemes}

The traditional kinetic mechanisms shown in Schemes 1 and 2 have been extensively used to describe Curtin-Hammett phenomena and are well known. Schemes 3, 4, and 5 describe situations involving equilibration between products. These represent worst-case scenarios from the point of dynamic kinetic resolution, as the final product ratios are solely dependent on these equilibria. Literature examples of successful dynamic kinetic resolution often involve the racemization of carbonyl substrates. A representative example is the preparation of $\alpha$-aminoesters from $\alpha$-haloesters in the presence of amines. ${ }^{3 \mathrm{~d}}$ Mechanistically, such a chemical process necessarily proceeds via enolic intermediates. Though experimental examples of dynamic kinetic resolution have been sufficiently described using Scheme 1, in fact the phenomenon is more adequately described by Scheme 6. The introduction of intermediate A in Scheme 6 between substrates $\mathrm{X}$ and $\mathrm{Y}$ accounts for this. The corresponding analytical solution resembles that given in eqs $1 \mathrm{a}-\mathrm{d}$ (see Supporting Information) and is consistent with experimental observations. Scheme 7 is a variant of Scheme 6 that combines direct and indirect connections between the substrates $\mathrm{X}$ and Y. Scheme 8 is a fuller representation of the transformations described by Noyori in his investigation of the hydrogenation of racemic mixtures of chiral esters. Since hydrogenation can take place via the re and si faces of the $\beta$-keto group, two possible products arise from each starting diastereomer. Hence, each of these transformations is associated with a different transition state and is thus treated as a separate elementary step. Schemes 9, 10, and 11 are examples of "crossover" product-forming schemes. Schemes 9 and 10 represent symmetric and asymmetric versions, respectively, of direct product-forming steps. Scheme 11 represents a hypothetical case involving combined direct product-forming steps and indirect product-forming steps passing through common intermediate I. Scheme 9 has been implicated in the hydride reduction of substituted cyclohexanones, ${ }^{18}$ the chlorodecarboxylation of cyclohexane- and cyclohexenecarboxylic acid derivatives, ${ }^{19}$ the isomerization of imines, ${ }^{20}$ and the transamination of imine derivatives. ${ }^{21}$ The final two schemes, 12 and 13, are double "feed-in" mechanisms considered previously by Seeman in his analytical investigations ${ }^{7 b}$ but are now investigated by the new approaches presented in this work.

\section{Determination of Key Parameters}

Once product ratio time profiles are determined, product excess time profiles can be immediately deduced from the simple relationship

$$
\operatorname{pe}(t)=\frac{\frac{[\mathrm{PX}]}{\mathrm{PY}]}-1}{\frac{[\mathrm{PX}]}{[\mathrm{PY}]}+1}
$$

where pe $(t)$ can represent enantiomeric or diastereomeric excess depending on the stereoisomeric relationship of the products. (See Scheme 2 for the case of racemizable enantiomeric substrate.) 


\section{SCHEME 2}

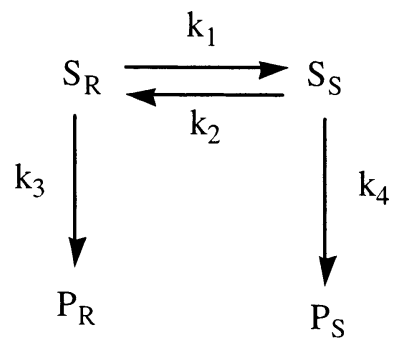

Figures $1 \mathrm{~A}$ and $\mathrm{B}$ and $2 \mathrm{~A}$ and $\mathrm{B}$ show simulations of the behavior of enantiomeric excesses with time as the magnitudes of the rate constant ratios are varied according to Scheme 2 for the case of reactions of racemic substrate mixtures. These curves were generated using eqs $1 \mathrm{c}$ and $\mathrm{d}$ and 2 with the condition $a$ $=b=1 .^{22}$ Experimentally generated curves can be obtained from quantitative product studies by monitoring the appearance of products with time. For direct comparison, rate constant ratios were chosen to match those used by Noyori in constructing his sigmoidal template curves using percent reaction completion as the independent variable. For example, Figure 1A corresponds directly to Figure 1e in ref 13a using identical sets of parameters. The shapes of the curves obtained here resemble exponential decays and approach minima as reactions approach $100 \%$ completion. The initial and final enantiomeric excesses may be easily determined either by extrapolation or by a nonlinear leastsquares analysis of kinetic data according to the complete timedependent function given in eq 2 . It will be shown vide infra that these two experimentally determined quantities along with

TABLE 1: Summary of Extended Kinetic Models Based on Scheme 1

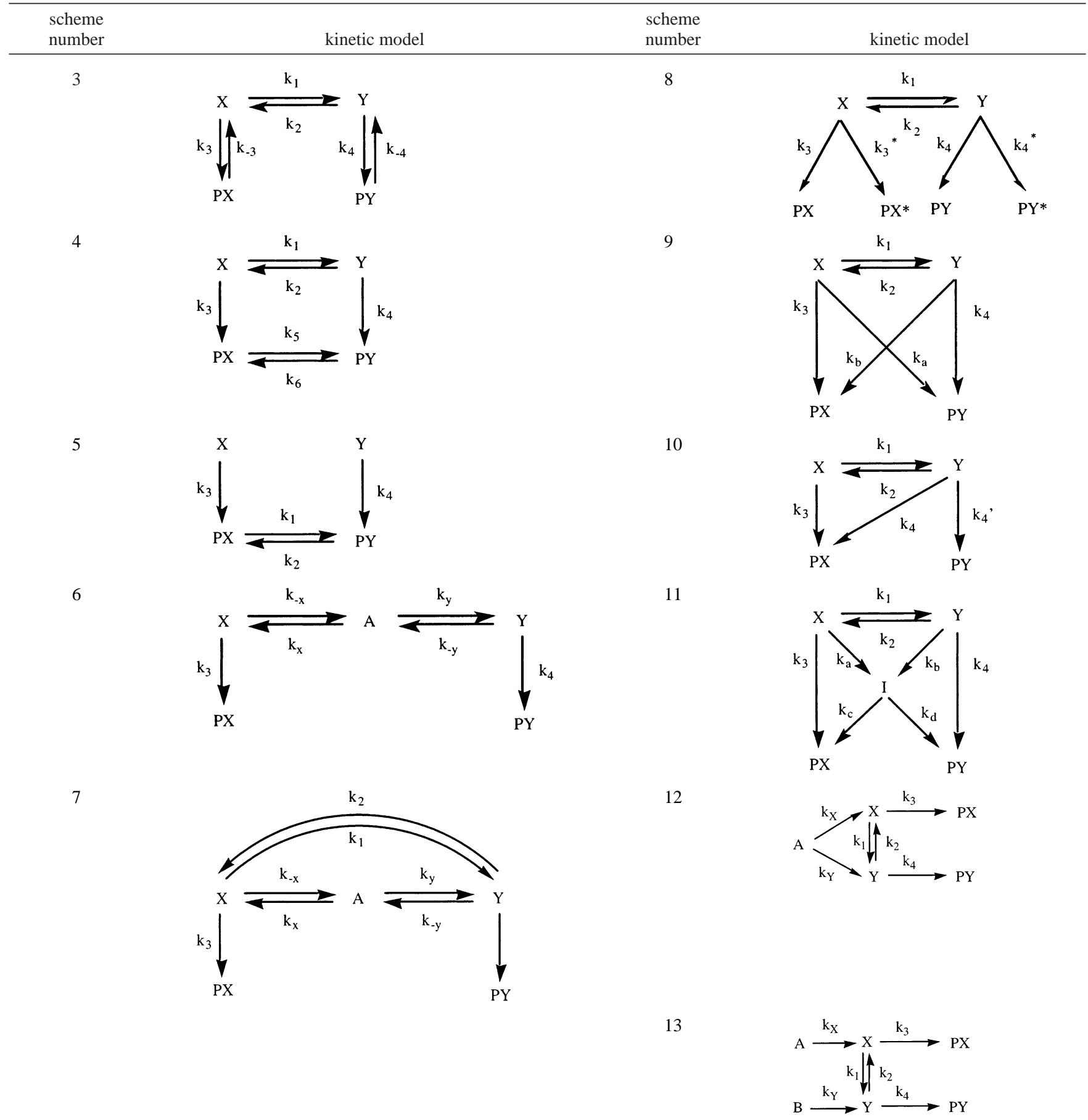



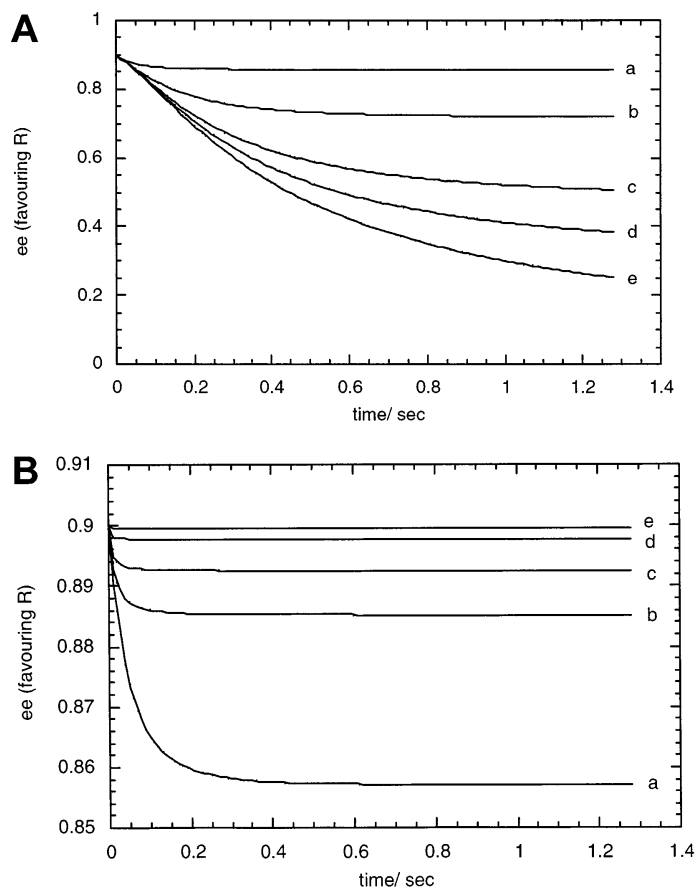

Figure 1. (a) Time dependence of the enantiomeric excess with respect to the $\mathrm{P}_{\mathrm{R}}$ product starting from a racemic mixture of substrates (Scheme 2). Simulated curves were constructed using eqs $1 \mathrm{c}$ and $\mathrm{d}$ and 2 with $a=b=1 ; k_{4}=1 ; k_{3} / k_{4}=95: 5 ; k_{1} / k_{3}=k_{2} / k_{3}=s ; \gamma 1+\gamma 2=k_{1}+$ $k_{2}+k_{3}+k_{4}=20+38 s$; and $\gamma 1 \gamma 2=k_{1} k_{4}+k_{2} k_{3}+k_{3} k_{4}=19+$ 380s: (a) $s=1$, (b) $s=0.2$, (c) $s=0.06$, (d) $s=0.03$, and (e) $s=$ 0.01. (b) Time dependence of the enantiomeric excess with respect to the $\mathrm{P}_{\mathrm{R}}$ product starting from a racemic mixture of substrates (Scheme 2). Simulated curves were constructed using eqs $1 \mathrm{c}$ and $\mathrm{d}$ and 2 with $a=b=1 ; k_{4}=1 ; k_{3} / k_{4}=95: 5 ; k_{1} / k_{3}=k_{2} / k_{3}=s ; \gamma 1+\gamma 2=k_{1}+$ $k_{2}+k_{3}+k_{4}=20+38 s$; and $\gamma 1 \gamma 2=k_{1} k_{4}+k_{2} k_{3}+k_{3} k_{4}=19+$ 380s: (a) $s=1$, (b) $s=3$, (c) $s=6$, (d) $s=20$, and (e) $s=100$.

the initial substrate ratio, $a / b$, are sufficient to determine relative energy barriers precisely and unambiguously for a chemical system obeying Scheme 1 or 2.

The entries in Table 2 summarize expressions pertaining to Scheme 1 for the product ratio and product excess at initial and final reaction times. These may be compared with the corresponding results given in Table S3 for other related schemes in Table 1. From Table S3, one can recognize a number of important patterns. In all cases except for Scheme 9, the initial product ratio is a linear function of the initial substrate ratio, $a / b$ :

$$
\frac{[\mathrm{PX}]_{0}}{[\mathrm{PY}]_{0}}=\text { intercept }+ \text { slope }\left(\frac{a}{b}\right)
$$

Although product concentrations are themselves zero at initial time, the corresponding product ratios are finite. The slope parameter in eq 3 is immediately related to the rate constant ratio $k_{3} / k_{4}$. All previous conformational studies on chemical systems obeying Scheme 1 have the common feature of estimating this target rate constant ratio from the final product ratio and the equilibrium constant for the interconverting conformers. Note that $\left([\mathrm{PX}]_{0} /[\mathrm{PY}]_{0}\right)=(a / b)\left(k_{3} / k_{4}\right)$ is identical to the Curtin-Hammett expression (vide infra) for the final product ratio when $K=(b / a)$. Equation 3 is a key relationship since it shows how $k_{3} / k_{4}$ can be determined directly without having to invoke either the Winstein-Holness equation or the Curtin-Hammett condition. Previous analytical solutions ${ }^{6,7}$ presented in the literature did not include expressions for the
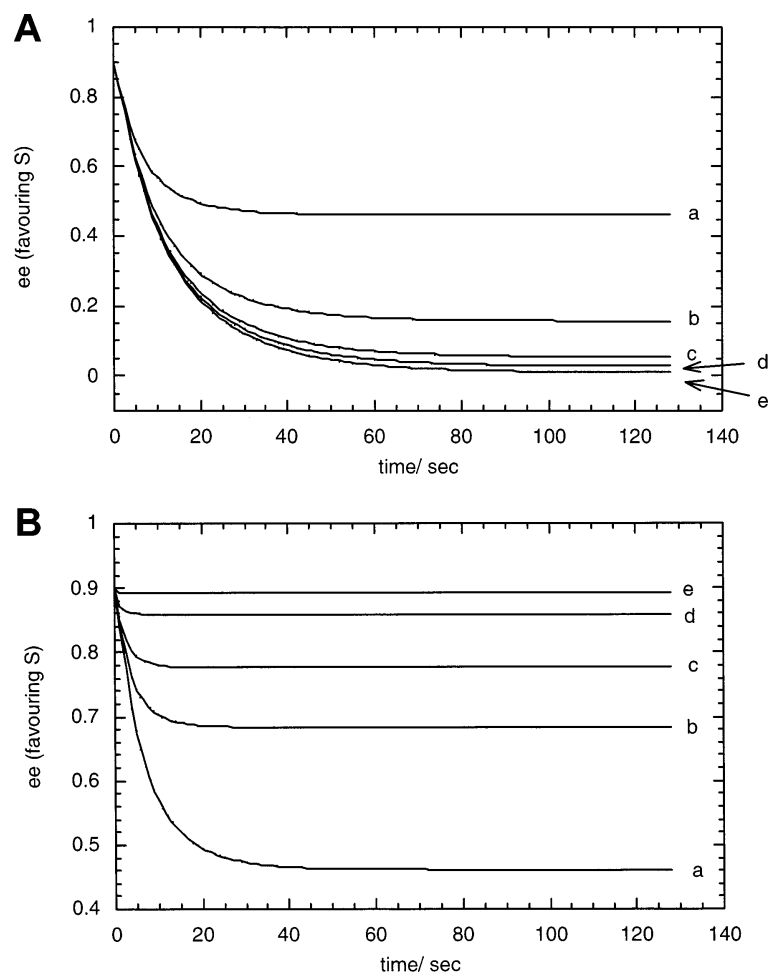

Figure 2. (A) Time dependence of the enantiomeric excess with respect to the $\mathrm{P}_{\mathrm{S}}$ product starting from a racemic mixture of substrates (Scheme 2 ). Simulated curves were constructed using eqs $1 \mathrm{c}$ and $\mathrm{d}$ and 2 with $a=b=1 ; k_{4}=1 ; k_{3} / k_{4}=5: 95 ; k_{1} / k_{3}=k_{2} / k_{3}=s ; \gamma 1+\gamma 2=k_{1}+$ $k_{2}+k_{3}+k_{4}=(20+2 s) / 19$; and $\gamma 1 \gamma 2=k_{1} k_{4}+k_{2} k_{3}+k_{3} k_{4}=(1$ $+20 s) /\left(19^{2}\right.$ ): (a) $s=1$, (b) $s=0.2$, (c) $s=0.06$, (d) $s=0.03$, and (e) $s=0.01$. (B) Time dependence of the enantiomeric excess with respect to the $\mathrm{P}_{\mathrm{S}}$ product starting from a racemic mixture of substrates (Scheme $2)$. Simulated curves were constructed using eqs $1 \mathrm{c}$ and $\mathrm{d}$ and 2 with $a=b=1 ; k_{4}=1 ; k_{3} / k_{4}=5: 95 ; k_{1} / k_{3}=k_{2} / k_{3}=s ; \gamma 1+\gamma 2=k_{1}+$ $k_{2}+k_{3}+k_{4}=(20+2 s) / 19 ;$ and $\gamma 1 \gamma 2=k_{1} k_{4}+k_{2} k_{3}+k_{3} k_{4}=(1$ $+20 s) /\left(19^{2}\right)$ : (a) $s=1$, (b) $s=3$, (c) $s=6$, (d) $s=20$, and (e) $s=$ 100 .

initial product ratio but rather were focused on obtaining approximate expressions for the final product ratio.

From Table S3, the majority of expressions for the final product ratio take the form

$$
\frac{[\mathrm{PX}]_{\infty}}{[\mathrm{PY}]_{\infty}}=\phi\left(\frac{k_{3}}{k_{4}}\right)
$$

where $\phi$ is some function of the initial substrate concentrations and all of the associated rate constants. Exceptions to this are mechanisms that have an equilibration step between products (see Schemes 4 and 5) and those that involve crossover productforming steps from both starting materials. (See Schemes 9, 10, and 11.) It is interesting that in comparing Schemes 12 and 13 the final product ratio is independent of the rate constants $k_{\mathrm{X}}$ and $k_{\mathrm{Y}}$ in the latter case, where $\mathrm{X}$ and $\mathrm{Y}$ originate from different precursors. However, in the former case, it is dependent on these feed-in rate constants when $\mathrm{X}$ and $\mathrm{Y}$ originate from a common precursor.

Equation 4 is significant as it provides a precise mathematical condition for the Curtin-Hammett limit. The final product ratio is dependent only on the energy barriers of elementary steps leading to the products and not on any prior equilibrium between substrates if and only if $\phi=1$. Normally, the Curtin-Hammett condition is represented by the two inequalities (i) $k_{1}, k_{2} \gg k_{3}$, $k_{4}$ and (ii) $k_{1} \approx k_{2}$. The $\phi=1$ condition is an equivalent but more compact statement of the principle. It should be mentioned 
TABLE 2: Summary of Initial and Final Product Ratios and Product Excesses for Scheme 1

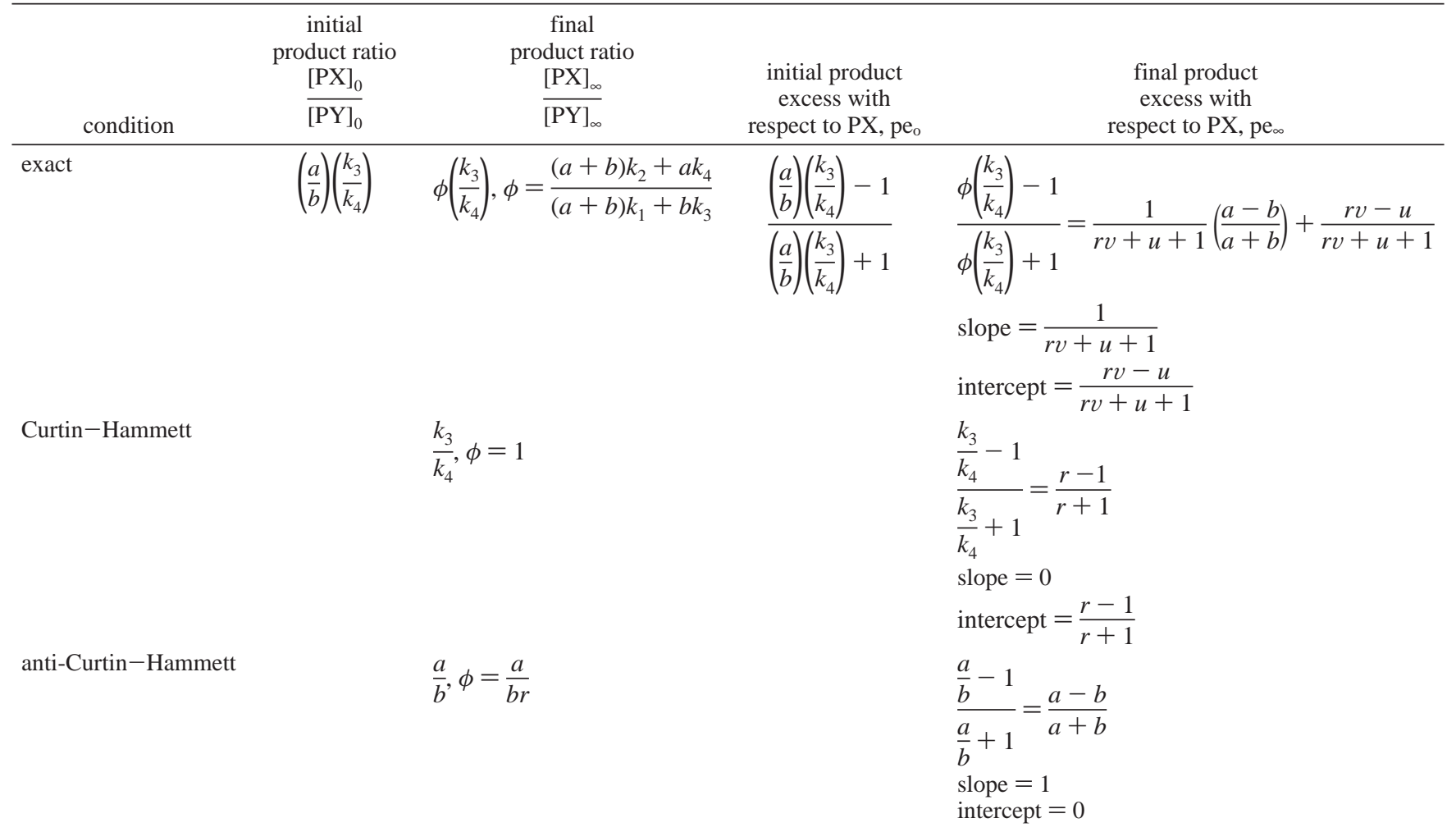

that both inequality conditions must apply so that $\phi=1$ and hence $\left([\mathrm{PX}]_{\infty} /[\mathrm{PY}]_{\infty}\right)=k_{3} / k_{4}$.

One can easily verify this by applying inequalities (i) and (ii) to the complete expression for $\phi$ pertaining to Scheme 1. Applying the first inequality alone results in a dependence of the final product ratio on the equilibrium between substrates: $\left([\mathrm{PX}]_{\infty} /[\mathrm{PY}]_{\infty}\right)=\left(k_{3} / k_{4}\right)\left(k_{2} / k_{1}\right)=\left(k_{3} / k_{4} K\right)$. This observation was noted by Seeman ${ }^{7}$ and resulted in a revision of the definition of this principle in IUPAC glossaries. ${ }^{23}$ In addition, the Zefirov expression $^{6}$ for the final product ratio when substrates are initially at equilibrium is verifiable at once from eq 4 when $b / a$ is replaced by $k_{1} / k_{2}$ in the expression for $\phi$ in Table 2 . Table $\mathrm{S} 4$ gives a complete summary of the resulting expressions for the final product ratios and excesses for more complicated schemes when the Curtin-Hammett condition is applied. For $\left([\mathrm{PX}]_{\infty} /[\mathrm{PY}]_{\infty}\right)=k_{3} / k_{4}$ to be true throughout, the standard inequality conditions need to be extended. For example, in Scheme $6, \phi=1$ is true if (i) $k_{\mathrm{X}}, k_{-\mathrm{X}}, k_{\mathrm{Y}}, k_{-\mathrm{Y}} \gg k_{3}, k_{4}$ and (ii) $k_{\mathrm{X}} \approx k_{-\mathrm{X}}$ and $k_{\mathrm{Y}} \approx k_{-\mathrm{Y}}$. Similar extended inequalities may be deduced for schemes given in Table 1. The forms of the limiting expressions in Tables 2 and S4 are reminiscent of Singleton's recent differential kinetic enantiomeric enhancement (DKEE) parameter. $^{12}$

Equation 4 also suggests a simple experiment to test the validity of the Curtin-Hammett condition for a particular chemical system. If a reaction obeying Scheme 1 is carried out at various temperatures with a fixed initial substrate ratio, then the dependence of the final product ratio on temperature can be determined. An Arrhenius or Eyring treatment of the data results in linear Arrhenius or Eyring plots if the CurtinHammett condition holds and nonlinear behavior if it does not. Nonlinearity occurs if $\phi$ in eq 4 is not equal to unity. The observation of nonlinearity requires care because such plots are rather robust to changes in curvature, so it is necessary to conduct experiments over as wide a temperature range as possible to observe any real nonlinear effects. A clear example of nonlinear behavior, however, has recently been reported for the reaction of triazolinediones with olefins in methanol solution involving biradical and zwitterionic intermediates. ${ }^{24}$ This reaction obeyed a mechanism that is a one-sided feed-in variant of Scheme 12.

Of particular note in Table S3 is a second general linear relationship found between the final product excess and the initial substrate excess, $(a-b) /(a+b)$ :

$$
\mathrm{pe}_{\infty}=\text { intercept }+ \text { slope }\left(\frac{a-b}{a+b}\right)
$$

The intercept and slope parameters are given by combinations of rate constant ratios of varying complexity, and $-1 \leq(a-$ $b) /(a+b) \leq 1$. Mechanisms involving an equilibration step between final products result in final product excesses that are independent of $(a-b) /(a+b)$. (See Schemes 3, 4, and 5.) Equation 5 is significant because it suggests for the first time, as will be shown in the following section, how all rate constant ratios can be determined entirely by experiment. In particular, two of the rate constant ratios that come out of this analysis are "cross" rate constant ratios since they connect one of the product-forming steps with either the forward or reverse steps in the equilibrium reaction. The determination of rate constant ratios immediately translates into the complete energy characterization of a chemical system. Moreover, the extent of dynamic kinetic resolution efficiency can also be experimentally determined from these rate constant ratio parameters. In previous analyses, the strategy employed whenever dynamic kinetic resolution was observed for a chemical system was to justify the validity of the Curtin-Hammett condition by carrying out separate molecular mechanics, semiempirical, or ab initio calculations to determine relative energy barriers for productforming steps and substrate equilibration steps. A notable example of this strategy is the recent work by Durst. ${ }^{3 \mathrm{~d}}$ The present conclusions demonstrate that this approach can be circumvented. 


\section{Characterization of Energetics and Graphical Analysis}

Applying eq 5 to Scheme 1 or 2 results in a slope and intercept of $1 /(r v+u+1)$ and $(r v-u) /(r v+u+1)$, respectively, where $r=k_{3} / k_{4}, u=k_{1} / k_{3}$, and $v=k_{2} / k_{3}$. The slope is always positive since $r, u$, and $v$ are positive quantities. The most common starting substrate conditions encountered in the synthesis of optically pure products are a racemic mixture $(a=b)$, pure $\mathrm{X}$ or $\mathrm{S}_{\mathrm{R}}(b=0)$, or pure $\mathrm{Y}$ or $\mathrm{S}_{\mathrm{S}}(a=0)$. The linear relationships given by eqs 3 and 5 suggest that product study experiments should be done under a common set of reaction conditions of temperature, pressure, catalyst species, and catalyst concentration for a range of initial substrate conditions beyond the above three cases. In this way, the rate constant ratio $r$ may be obtained from the slope of $[\mathrm{PX}]_{0} /[\mathrm{PY}]_{0}$ versus $a / b$, whereas the rate constant ratios $u$ and $v$ may be determined from the slope and intercept parameters determined from eq 5 according to

$$
\begin{aligned}
& u=\frac{1}{2}\left[\frac{1-\text { intercept }}{\text { slope }}-1\right] \\
& v=\frac{1}{2 r}\left[\frac{1+\text { intercept }}{\text { slope }}-1\right]
\end{aligned}
$$

Equations 6a and b readily yield the equilibrium constant $K$ : $K$ $=k_{1} / k_{2}=u / v$. Similar strategies can be used to obtain the rate constant ratios $r, u$, and $v$ for Schemes $6,7,12$, and $13 ; r, k_{3} * /$ $k_{4}{ }^{*}, k_{3} / k_{3} *, w, u$, and $v$ for Scheme $8 ; r, u, v, k_{\mathrm{a}} / k_{4}$, and $k_{\mathrm{b}} / k_{4}$ for Scheme 9; and $r, r^{\prime}, u$, and $v$ for Scheme 10. In the case of Scheme 8, which is relevant to the chemical examples of Noyori, the linear property between the final product excess and the initial substrate excess exists when all products arising from one starting stereoisomer are compared with all products arising from the other. Hence, for Scheme 8, pe $e_{\infty}$ in eq 5 is defined as $\mathrm{pe}_{\infty}=\left\{\left([\mathrm{PX}]+\left[\mathrm{PX}^{*}\right]\right)-([\mathrm{PY}]+[\mathrm{PY} *])\right\} /\left\{\left([\mathrm{PX}]+\left[\mathrm{PX}^{*}\right]\right)\right.$ $+([\mathrm{PY}]+[\mathrm{PY} *])\}$. Individual pairwise product excesses such as $([\mathrm{PY}]-[\mathrm{PY} *]) /([\mathrm{PY}]+[\mathrm{PY} *])$ are not linear in the initial substrate excess $(a-b) /(a+b)$.

The results for Scheme 9 are significant since Seeman notes 25 that the application of the Winstein-Holness treatment cannot yield unique solutions of rate constants for this Scheme. In the present treatment, $r, k_{\mathrm{a}} / k_{4}$, and $k_{\mathrm{b}} / k_{4}$ are obtained directly from a plot of the initial product ratio against $a / b$. The remaining two ratios $u$ and $v$ are obtained from a straightforward algebraic treatment of the slope and intercept of a plot of the final product excess against $(a-b) /(a+b)$ once the previous three ratios are known. In the case of Scheme 11, however, it is impossible to implement this strategy unless separate kinetic experiments are performed to determine at least two absolute rate constants.

Parts A and B of Figure 3 show example energy reaction progress diagrams for the case of enantiomeric substrates (see Scheme 2) reacting to produce diastereomeric products in the presence of chiral reagents under anti-Curtin-Hammett and Curtin-Hammett conditions, respectively. The correspondences between energy differences and rate constant ratios defined in this investigation are shown. From the slope and intercept parameters of eq 5, one may apply two limiting conditions corresponding to the Curtin-Hammett limit and the antiCurtin-Hammett limit, respectively. In terms of $r, u$, and $v$, these conditions become $u=v \rightarrow \infty$ and $u=v \rightarrow 0$, respectively. It is straightforward to show that these limiting situations correspond directly to the case when the barrier for the equilibration step is far smaller than those for the product-
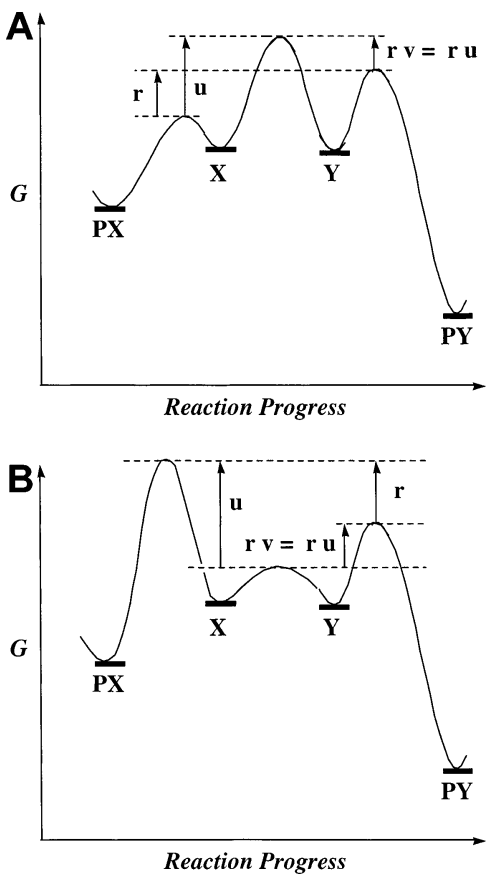

Figure 3. (A) Energy-reaction progress diagram showing correspondence between rate constant ratios and their respective energy differences for the anti-Curtin-Hammett case. This diagram is specific to the case of enantiomeric substrates $\mathrm{X}$ and $\mathrm{Y}$ leading to diastereomeric products PX and PY via chiral reagents. (B) Energy-reaction progress diagram showing correspondence between rate constant ratios and their respective energy differences for the Curtin-Hammett case. This diagram is specific to the case of enantiomeric substrates $\mathrm{X}$ and $\mathrm{Y}$ leading to diastereomeric products $\mathrm{PX}$ and PY via chiral reagents.

forming steps for the Curtin-Hammett limit and vice versa for the anti-Curtin-Hammett limit. In the Curtin-Hammett limit, the slope of eq 5 tends to zero, and the intercept tends to ( $r-$ $1) /(r+1)$, whereas in the anti-Curtin-Hammett limit the slope tends to unity and the intercept tends to zero. Table S4 summarizes the results of imposing these two limiting conditions on all schemes given in Table 1. It is observed that the patterns described hold up for several variants of Scheme 1.

Figure 4A shows the result of a plot of the final product excess, $\mathrm{pe}_{\infty}$, versus the initial substrate excess, $(a-b) /(a+b)$, according to eq 5 with fixed $r$ (with $r>1$ ) and varying $u=v$ for the case of enantiomeric substrates. Absolute values have not been used in the definitions of excesses so that continuous straight lines can be drawn. The connection between Noyori's previous analysis and this plot is that Noyori's product study data correspond to a single point determination, namely, the product excess obtained for an initial racemic substrate mixture $((a-b) /(a+b)=0)$. The limiting Curtin-Hammett condition given by the horizontal line indicates that the final product ratio will always tend to the same value regardless of the substrate ratio composition. This corresponds to the most efficient dynamic kinetic resolution possible. The horizontal line is near the top of the plot toward the vertex labeled PX. This is consistent with the fact that $r>1$ (or $k_{3}>k_{4}$ ), making product PX the major product of the reaction. However, the anti-CurtinHammett condition given by the diagonal line of unit slope and zero intercept indicates that the final product ratio exactly matches the initial substrate ratio. This corresponds to kinetic resolution with no dynamic component. Such anti-CurtinHammett behavior has been observed for the reduction of sterically hindered $\beta$-diketones. ${ }^{26}$ 


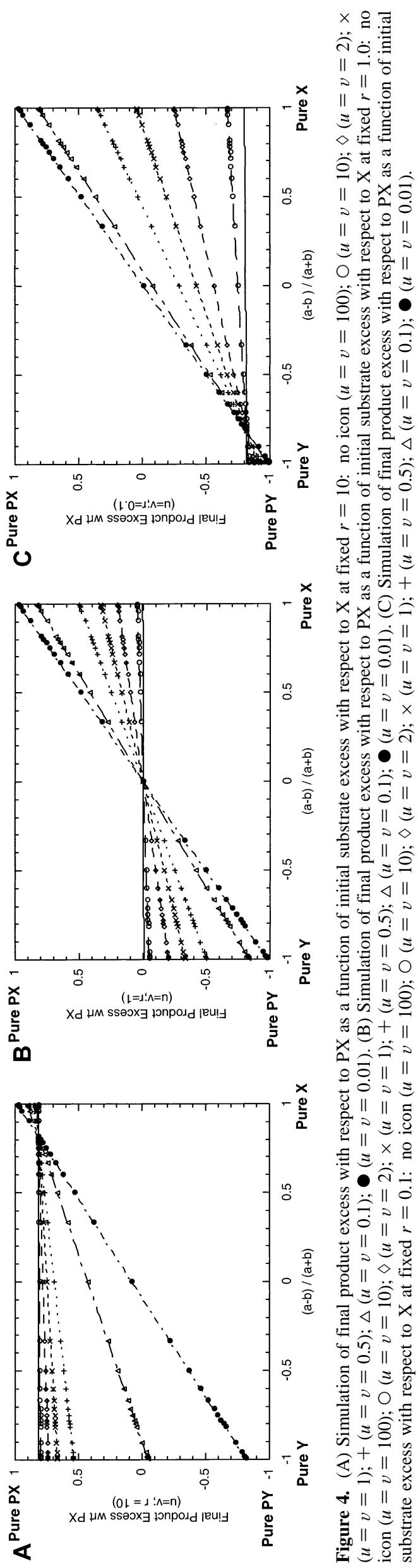

TABLE 3: Summary of Dynamic Kinetic Resolution Efficiency Factors for Various Kinetic Models ${ }^{a}$

\begin{tabular}{|c|c|c|}
\hline $\begin{array}{l}\text { scheme } \\
\text { number }\end{array}$ & $\begin{array}{l}\text { dynamic kinetic } \\
\text { resolution efficiency } \\
\text { factor, } \epsilon_{\mathrm{DKR}}\end{array}$ & $\begin{array}{l}\text { optimum initial } \\
\text { substrate excess with } \\
\text { respect to } \mathrm{X}, \mathrm{se}_{\mathrm{o}}{ }^{* b}\end{array}$ \\
\hline \multirow[t]{2}{*}{1} & $r v+u$ & $r-1$ \\
\hline & $\overline{r v+u+1}$ & $\overline{r+1}$ \\
\hline 3 & not applicable & not applicable \\
\hline 4 & not applicable & not applicable \\
\hline 5 & not applicable & not applicable \\
\hline \multirow[t]{2}{*}{6} & $r v+u$ & $r-1$ \\
\hline & $\overline{r v+u+1}$ & $\overline{r+1}$ \\
\hline \multirow[t]{2}{*}{7} & $r v+u$ & $r-1$ \\
\hline & $\overline{r v+u+1}$ & $\overline{r+1}$ \\
\hline \multirow[t]{2}{*}{8} & $w u+v$ & $1-w$ \\
\hline & $\overline{w u+v+w}$ & $\overline{1+w}$ \\
\hline \multirow[t]{2}{*}{9} & $1-\frac{A_{2}}{B_{2}}$ & $\frac{k_{\mathrm{a}}-k_{\mathrm{b}}}{k_{4}}+r-1$ \\
\hline & & $\overline{\frac{k_{\mathrm{a}}+k_{\mathrm{b}}}{k_{4}}+r+1}$ \\
\hline \multirow[t]{2}{*}{10} & $u\left(r+r^{\prime}\right)+v r r^{\prime}$ & $r^{\prime}(r-1)-r$ \\
\hline & $\overline{(1+u)\left(r+r^{\prime}\right)+v r r^{\prime}}$ & $\overline{r^{\prime}(r+1)+r}$ \\
\hline \multirow[t]{2}{*}{11} & $1-\frac{C_{2}}{D_{2}}$ & $\frac{k_{\mathrm{a}}-k_{\mathrm{b}}}{k_{4}}+r-1$ \\
\hline & & $\overline{\frac{k_{\mathrm{a}}+k_{\mathrm{b}}}{k_{4}}+r+1}$ \\
\hline \multirow[t]{2}{*}{$12^{c}$} & $r v+u$ & $\underline{r-1}$ \\
\hline & $\overline{r v+u+1}$ & $\overline{r+1}$ \\
\hline \multirow[t]{2}{*}{13} & $r v+u$ & $\underline{r-1}$ \\
\hline & $\overline{r v+u+1}$ & $\overline{r+1}$ \\
\hline
\end{tabular}

${ }^{a}$ Variable definitions are given in Tables S3 and S4. ${ }^{b}$ Corresponds to a value of $\mathrm{se}_{0} *$ yielding a $\mathrm{pe}_{\infty}$ obtained under Curtin-Hammett conditions. ${ }^{c}$ For this case, $\mathrm{pe}_{\infty}$ is plotted against $\frac{k_{\mathrm{X}}-k_{\mathrm{Y}}}{k_{\mathrm{X}}+k_{\mathrm{Y}}}$.

\section{Optimization of Dynamic Kinetic Resolution}

The Curtin-Hammett principle and dynamic kinetic resolution are intimately connected, as one implies the other. The more efficiently dynamic kinetic resolution is achieved in a chemical system, the more it is true that the Curtin-Hammett condition is valid. The variation of the slope in eq 5 between zero and unity suggests that a dynamic kinetic resolution efficiency factor may be defined between these two extremes. A slope of zero corresponds to $100 \%$ efficient dynamic kinetic resolution, and a slope of unity corresponds to $0 \%$ efficiency. In terms of the rate constant parameters $r, u$, and $v$, the dynamic kinetic resolution efficiency parameter for Schemes 1 and 2 is then given by

$$
\epsilon_{\mathrm{DKR}}=\frac{r v+u}{r v+u+1}
$$

where $0 \leq \epsilon_{\mathrm{DKR}} \leq 1$. Table 3 summarizes the expressions for this parameter pertaining to all schemes given in Table 1. Note that for Schemes 3, 4, and 5 the parameter $\epsilon_{\mathrm{DKR}}$ is not applicable since the final product excess is independent of the initial substrate excess. From the variation exhibited in Figure 4A, it is observed that all lines intersect at the point $((r-1) /(r+1)$, $(r-1) /(r+1))$. The intersection corresponds to the initial substrate ratio required to effect a final product ratio that would result under the condition of $100 \%$ dynamic kinetic resolution. Table 3 also includes expressions for these optimum initial 
substrate ratios for each of the schemes given in Table 1. The

simulation depicted in Figure 4A with fixed $r$ and varying $u=$

$v$ suggests how manipulating the prior substrate equilibrium can

optimize a reaction with fixed product-forming reactions. This strategy corresponds exactly to the technique of dynamic thermodynamic resolution described by Beak. ${ }^{4}$ The starting substrate ratio is biased in favor of the product of interest by shifting the equilibrium in the direction of the substrate that leads to that product. This is commonly done by manipulating the temperature of the equilibrium reaction before the productforming reactions are carried out. Alternatively, the starting materials, usually obtained as products from a previous step, can be separated, remixed according to the optimum ratio, and then allowed to react under conditions leading to the desired products. This second option may be advantageous if the starting materials are a diastereomeric pair rather than an enantiomeric pair. In either case, selecting a target initial substrate excess equal to $(r-1) /(r+1)$ takes the guesswork out of choosing an appropriate substrate condition to maximize the yield of the desired product. Figure $4 \mathrm{~B}$ and $\mathrm{C}$ shows similar simulations under the conditions of $r=1$ and $r<1$, respectively. The case of $r=1$ corresponds to equal energy barriers for the productforming reactions and an intersection of $(0,0)$ corresponds to an initial racemic substrate mixture leading to a final optimal racemic product mixture. The case $r<1$ is analogous to that shown in Figure 4A except that product PY is now favored, hence the horizontal Curtin-Hammett line and intersection point appear in the lower left quadrant of the graph.

The simulations shown in Figure 5A-C describe the opposite variation shown in Figure $4 \mathrm{~A}-\mathrm{C}$, namely, that $u=v$ is fixed and $r$ is varied. This corresponds to the more common optimization in which the product-forming reactions are manipulated. In Figure 5A with $u=v$ exceeding unity, all lines are nearly horizontal so that the chemical system is predisposed to being Curtin-Hammett-like. As expected, products PX and $\mathrm{PY}$ are favored when $r>1$ (or $k_{3}>k_{4}$ ) and $r<1$ (or $k_{3}<k_{4}$ ), respectively. As $u=v$ becomes substantially less than unity, the chemical system shifts to being anti-Curtin-Hammett-like with all lines tending to have a slope of unity and passing through the origin. Product PX is favored if an initial substrate excess is chosen in favor of $X$ (upper right quadrant), whereas product PY is favored if an initial substrate excess is chosen in favor of Y (lower left quadrant).

The advantage of constructing experimental plots according to eq 5 is that they show how far a reaction can be pushed in the direction of $100 \%$ efficient dynamic kinetic resolution. Hence, a third strategy applicable to all graphs shown is that once the energetics of a reaction are determined reaction conditions may be selected so as to make the experimentally determined line more horizontal, that is, to make the chemical system behave in a more Curtin-Hammett-like manner. Examples of achieving this, as demonstrated by Noyori's results shown in Table 4, include changing the solvent of the reaction, adding appropriate catalysts, changing the type of catalyst used, or changing the concentration of catalyst used.

An alternative approach to optimizing $\epsilon$ is to vary the temperature of the reaction. It is readily apparent that each of the rate constant ratio parameters in eq 7 scales with temperature in an Arrhenius fashion. It can be shown that the variation of $\epsilon$ with $T$ is sigmoidal where two cases arise depending on the signs of the energy differences between product-forming steps and either the forward or reverse step in the equilibrium. In the first case, a sigmoidal graph with a maximum asymptote equal to $\epsilon=1$ at $T=0$ and a minimum asymptote equal to $\epsilon=C<$
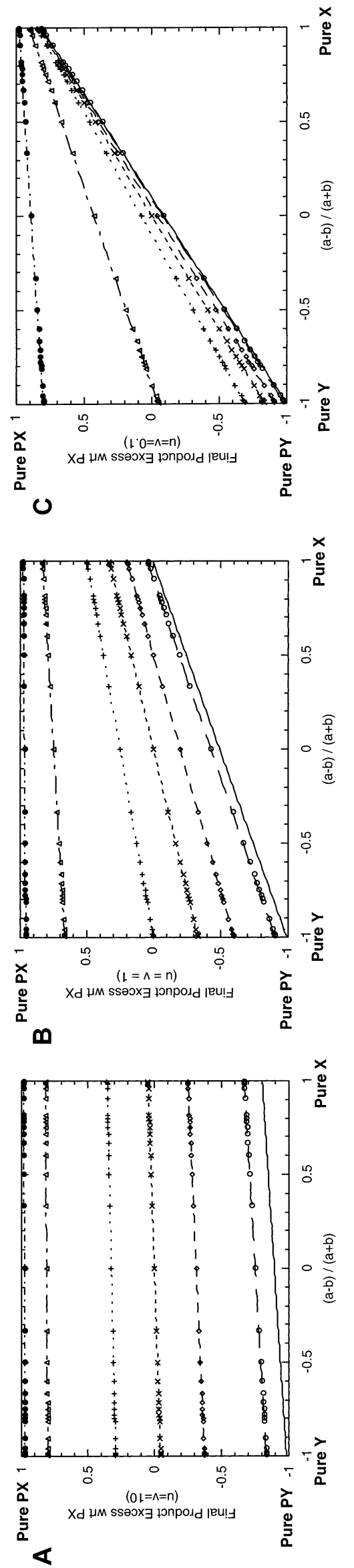

㞼 II 
TABLE 4: Reevaluation of Noyori's Parameters for the Hydrogenation of Various $\beta$-Ketoesters using BINAP-Ru(II) Catalysts On the Basis of the Rate Constant Definitions Given in Scheme 8 in Table $1^{a}$

\begin{tabular}{|c|c|c|c|c|c|c|c|}
\hline $\begin{array}{c}\text { racemic } \\
\text { substrate, } a=b\end{array}$ & $\begin{array}{l}\text { key reaction } \\
\text { conditions }\end{array}$ & $\frac{1}{w}^{b}$ & $\begin{array}{c}\frac{k_{1}}{k_{3}+k_{3}^{\prime}}=\frac{k_{2}}{k_{3}+k_{3}^{\prime}} \\
(u=v)^{c}\end{array}$ & $\left|e e_{\mathrm{p}}^{\infty}\right|^{d}$ & $\phi^{e}$ & $\begin{array}{l}\text { dynamic resolution } \\
\text { efficiency factor, }{ }^{f} \\
\epsilon_{\text {DKR }} \\
\end{array}$ & $\begin{array}{l}\text { selectivity } \\
\text { parameter, } \\
\quad \text { SEL }_{\infty} \\
\end{array}$ \\
\hline $\mathrm{OCH}_{3}$ & $\begin{array}{l}\mathrm{CH}_{2} \mathrm{Cl}_{2} \\
a=b=2.7 \mathrm{M} \\
{[(R) \text { Binap }-\mathrm{Ru}(\mathrm{II})]} \\
=2.6 \mathrm{mM}\end{array}$ & 9.8 & 0.45 & 0.68 & 0.53 & 0.83 & 0.82 \\
\hline & $\begin{array}{l}\mathrm{CH}_{3} \mathrm{OH} \\
a=b=2.7 \mathrm{M} \\
{[(R) \text { Binap }-\mathrm{Ru}(\mathrm{II})]} \\
=2.6 \mathrm{mM}\end{array}$ & 5.9 & 0.24 & 0.44 & 0.44 & 0.62 & 0.57 \\
\hline $\mathrm{CH}_{3}$ & $\begin{array}{l}\mathrm{CH}_{2} \mathrm{Cl}_{2} \\
a=b=2.7 \mathrm{M} \\
{[(R) \operatorname{Binap}-\mathrm{Ru}(\mathrm{II})]} \\
=2.6 \mathrm{mM}\end{array}$ & 0.067 & 92 & 0.87 & 1.08 & 0.99 & 0.93 \\
\hline $\mathrm{HCOC}_{6} \mathrm{H}_{5}$ & $\begin{array}{l}\mathrm{CH}_{2} \mathrm{Cl}_{2} \\
a=b=2.7 \mathrm{M} \\
{[(R) 3,5-x y l y \mathrm{BINAP}-\mathrm{Ru}(\mathrm{II})]} \\
=2.6 \mathrm{mM}\end{array}$ & 0.038 & 94 & 0.92 & 1.13 & 0.99 & 0.96 \\
\hline$\underset{\mathrm{OCH}_{3}}{-\mathrm{OCH}_{3}}$ & $\begin{array}{l}\mathrm{CH}_{3} \mathrm{OH} \\
a=b=0.33 \mathrm{M} \\
{[(R) \mathrm{BINAP}-\mathrm{Ru}(\mathrm{II})]} \\
=0.18 \mathrm{mM}\end{array}$ & 13 & 0.88 & 0.79 & 0.67 & 0.93 & 0.89 \\
\hline $\mathrm{Br}$ & $\begin{array}{l}\mathrm{CH}_{3} \mathrm{OH} \\
a=b=0.33 \mathrm{M} \\
{[(R) \mathrm{BINAP}-\mathrm{Ru}(\mathrm{II})]} \\
=0.65 \mathrm{mM}\end{array}$ & 13 & 0.22 & 0.66 & 0.35 & 0.75 & 0.82 \\
\hline
\end{tabular}

${ }^{a}$ Data taken from reference $13 \mathrm{~b} .{ }^{b}$ Corresponds to $\frac{k_{\mathrm{R}}}{k_{\mathrm{S}}}$ in Noyori's notation. ${ }^{c}$ Corresponds to $\frac{k_{\text {inv }}}{k_{\mathrm{R}}}$ and $k_{1}=k_{2}=k_{\text {inv }}$ in Noyori's notation. ${ }^{d}$ Evaluated according to $\left|e e_{\mathrm{p}}^{\infty}\right|=\left|\frac{v-w u}{w u+v+w}\right|=\left|\frac{u(1-w)}{u(1+w)+w}\right|$ where $u=v$ and $\mathrm{ee}_{\mathrm{s}}{ }^{\mathrm{o}}=0$ since the initial substrate is racemic. ${ }^{e}$ Evaluated according to $\phi=\frac{2 u+w}{2 u+1}$ where $u=v$ and $a=b$ since the initial substrate is racemic. ${ }^{f}$ Evaluated according to $\epsilon_{\mathrm{DKR}}=\frac{w u+v}{w u+v+w}=\frac{u(1+w)}{u(1+w)+w}$ where $u=v \cdot{ }^{g}$ Noyori's selectivity parameter defined as $\mathrm{SEL}_{\infty}=\left(1+\frac{k_{4}}{k_{3} \phi}+\frac{k_{3}{ }^{*}}{k_{3}}+\frac{k_{4}{ }^{*}}{k_{3} \phi}\right)^{-1}=\left(\frac{k_{3}}{k_{3}+k_{3}{ }^{*}}\right)\left(\frac{2 u+w}{2[u(1+w)+w]}\right)$ in present notation.

1 in the high-temperature limit applies when $E 4-E 2>0$ and $E 3-E 1>0$, or $E 4-E 2>0$ and $E 3-E 1<0$, or $E 4-E 2$ $<0$ and $E 3-E 1>0$, where $C$ is a constant composed of Arrhenius preexponential factors $[(A 2 / A 4)+(A 1 / A 3)] /[(A 2 / A 4)$ $+(A 1 / A 3)+1]$. In this case, decreasing temperature maximizes $\epsilon$ so that $C \leq \epsilon \leq 1$, where $\epsilon=C$ represents a minimum value for the efficiency parameter. In the second case, a sigmoidal graph with a minimum asymptote equal to $\epsilon=0$ at $T=0$ and a maximum asymptote equal to $\epsilon=C$ in the high-temperature limit applies when $E 4-E 2<0$ and $E 3-E 1<0$. In this case, increasing temperature maximizes $\epsilon$ so that $0 \leq \epsilon \leq C$, where $\epsilon=C$ is now a maximum value for the efficiency parameter. This situation corresponds uniquely to the antiCurtin-Hammett condition in which optimization is affected largely by entropic factors. Clearly, practical considerations governing the solvent of the reaction and the solubilities of reaction components will dictate whether such a strategy can be implemented in a given chemical system.

\section{Connections with Literature Examples}

The literature shows that the number of quantitative studies carried out to obtain reliable estimates of energy barriers in Curtin-Hammett schemes lags far behind the number of chemical examples found that conform to any one of the schemes shown in Table 1. At present, the few examples available that can be used to test ideas put forward in this work are discussed below.

(I) Stereochemically Related Substrates. A reevaluation of Durst's results for the reaction of racemic mixtures of diaster- eomeric $\alpha$-halopantolactones with benzylamine according to the present treatment yields lower-limit estimates for $r$ according to Scheme 1. For example, his observed product ratio of $\left([\mathrm{PX}]_{\infty} /\right.$ $\left.[\mathrm{PY}]_{\infty}\right)=7$ translates into the exact relation $\left([\mathrm{PX}]_{\infty} /[\mathrm{PY}]_{\infty}\right)=$ $(2 v r+1) /(2 u+1)$. Since the substrates are diastereomeric, $u$ and $v$ have different values. It is readily apparent that an infinite number of combinations of $u$ and $v$ are possible so that ([PX $]_{\infty} /$ $\left.[\mathrm{PY}]_{\infty}\right)=7$ or $r=(3+7 u) / v$. A graphical analysis of the latter relation for positive $u$ and $v$ indicates that it has a minimum value of 7 when $u$ and $v$ tend to infinity, that is, in the CurtinHammett limit. This means that the energy difference between steps 3 and 4 is at least $1.2 \mathrm{kcal} / \mathrm{mol}$ at $300 \mathrm{~K}$ in this example, as noted by Durst. ${ }^{3 \mathrm{~d}}$ This estimate, however, does not give any information as to how this energy barrier difference compares with that for the racemization of substrates. Durst relied on theoretical calculations of transition states for product-forming steps and racemization steps to justify that the Curtin-Hammett condition was applicable to the chemical system investigated. The present investigation offers the possibility of obtaining all three rate constant ratios so that the dynamic kinetic resolution that is observed can be quantified and then improved upon.

Relationships for Scheme 8 given in Tables S4 and 3 and Noyori's rate constant ratio data determined by the method described above (see Introduction) were used to reevaluate his previous experimental data for the hydrogenation of $\beta$-ketoesters using BINAP catalysts. ${ }^{13 \mathrm{~b}}$ The resulting efficiency factors using eq 7 are summarized in Table 4. For comparison, Noyori's selectivity parameter, SEL, is included in the compilation. In all cases, $\epsilon_{\mathrm{DKR}}$ exceeded about 0.6 so that the dynamic kinetic 
resolution was better than $60 \%$ efficient, thereby confirming that the Curtin-Hammett condition was indeed operative. For the pairwise comparisons shown, the trends in $\epsilon_{\mathrm{DKR}}$ and SEL parallel one another. This is satisfying since Noyori's investigation is presently the best quantitative work available on dynamic kinetic resolution. A reinvestigation of Noyori's chemical systems by the graphical method presented in this work may offer firmer estimates of relative energy barriers.

Equation 7 can further be used to evaluate the efficiency of the dynamic kinetic resolution of the 1,1-difluoro-2,3-dimethylcyclopropane system studied by Dolbier and Enoch. ${ }^{27}$ The energetics of the pyrolytic extrusion of difluorocarbene leading to cis- and trans-1,2-dimethylethylene products was studied in the gas phase. Using estimates of all four rate constants given in their work at $297 \mathrm{~K}$ results in an efficiency of $98 \%$ for that system. This is consistent with the observation that the major product is the trans olefin, which in turn is formed from the more abundant trans-1,1-difluoro-2,3-dimethyl-cyclopropane at equilibrium.

(II) Conformationally Related Substrates. The problem of quantifying Curtin-Hammett efficiencies is more challenging when dealing with reactions of conformationally related substrates. Here, control over substrate ratios is not as facile as in the case of stable stereoisomeric substrates. The usual strategy is first to determine the equilibrium constant, $K$, and the final product ratio, $R_{\infty}$, in separate experiments in the absence and in the presence of reagents leading to products, respectively. Then, a global first-order observed rate constant, $k_{\mathrm{WH}}$, is obtained from the kinetic profile for the appearance of total product. The ratio $k_{3} / k_{4}$ is then obtained using all three parameters through the Winstein-Holness equation, assuming that the CurtinHammett approximation is valid. An example chemical system where this paradigm has been employed is the $N$-methylation of pyrrolidines studied by Seeman ${ }^{25,28}$ in which estimates of $K$ and $R_{\infty}$ were made by NMR measurements. The above parameters are, however, insufficient to determine precisely the efficiency parameter as defined by eq 7. First, only lower-limit estimates of the equilibrium constant, $K$, are given. Second, estimates of $r$ or $k_{3} / k_{4}$ hinge on the assumption that the Winstein-Holness and Curtin-Hammett conditions apply to the system (which is what is being tested). Third, estimates of any one of the key cross rate constant ratios $k_{1} / k_{3}, k_{2} / k_{3}, k_{1} / k_{4}$, or $k_{2} / k_{4}$ are lacking. Last, the conductometric technique employed allowed only the appearance of total product to be monitored kinetically. Hence, the time evolution of individual products could not be determined by this method.

Despite these limitations, lower-limit estimates of the efficiencies of product resolutions can still be made for these chemical systems. Since observed kinetics experiments are usually conducted from equilibrium mixtures of substrates and observed pseudo-first-order single-exponential kinetic curves are recorded for the appearance of total product, it can be assumed that the Winstein-Holness equation is valid (see Appendix). To test the validity of the Curtin-Hammett approximation, however, the exact relationship for the final product ratio under these conditions must be used. Hence, we have according to Scheme 1

$$
R_{\infty}=\frac{[\mathrm{PX}]_{\infty}}{[\mathrm{PY}]_{\infty}}=\frac{k_{3}\left[k_{2}(1+K)+k_{4}\right]}{K k_{4}\left[k_{2}(1+K)+k_{3}\right]}
$$

for the final product ratio. If estimates of the initial product ratios were made, then the exact relation $R_{0}=\left([\mathrm{PX}]_{0} /[\mathrm{PY}]_{0}\right)=$ $a k_{3} / b k_{4}$ can be used to obtain the following expressions for $k_{3}$ and $k_{4}$ from the Winstein-Holness equation:

$$
\begin{gathered}
k_{3}=\frac{k_{\mathrm{WH}} R_{0}(K+1)}{1+R_{0}} \\
k_{4}=\frac{k_{\mathrm{WH}}(K+1)}{K\left(1+R_{0}\right)}
\end{gathered}
$$

$k_{\mathrm{WH}}$ is the observed first-order rate constant for the appearance of total product. Comparing these expressions with those derived by Seeman shows that here $R_{0}$ replaces $R_{\infty}$, where $R_{\infty}$ is approximated by the Curtin-Hammett relation $R_{\infty}=\left(k_{3} / k_{4} K\right)$ $=R_{0}$. Substituting eqs $9 \mathrm{a}$ and $\mathrm{b}$ into eq 8 yields an expression for $k_{2}$ :

$$
k_{2}=\frac{k_{\mathrm{WH}} R_{0}\left(1-R_{\infty} K\right)}{K\left(1+R_{0}\right)\left(R_{\infty}-R_{0}\right)}
$$

Furthermore, since $k_{1}=k_{2} K$,

$$
k_{1}=\frac{k_{\mathrm{WH}} R_{0}\left(1-R_{\infty} K\right)}{\left(1+R_{0}\right)\left(R_{\infty}-R_{0}\right)}
$$

Substituting all expressions for $k_{1}, k_{2}, k_{3}$, and $k_{4}$ into eq 7 finally yields the Curtin-Hammett efficiency factor

$$
\epsilon_{\mathrm{DKR}}=\frac{\left(1-R_{\infty} K\right)\left(1+R_{0}\right)}{\left(1-R_{\infty} K\right)\left(1+R_{0}\right)+(K+1)\left(R_{\infty}-R_{0}\right)}
$$

If $R_{\infty}$ is replaced by its Curtin-Hammett approximation, which is identical to $R_{0}$, then $\epsilon=1$, meaning that the resolution is $100 \%$ efficient as expected. A graphical analysis of eq 12 with $R_{0}$ as an independent variable shows that it has a hyperbolic form with a discontinuity at $R_{0}=1 / K$. There are two cases to consider that satisfy the meaningful condition of $0 \leq \epsilon \leq 1$, namely, $R_{0}<R_{\infty}<1 / K$ and $R_{0}>R_{\infty}>1 / K$. The former case results in a concave-up curve between zero and $1 / K$ with a minimum efficiency equal to $\left(1-R_{\infty} K\right) /\left(1+R_{\infty}\right)$ at $R_{0}=0$, whereas the latter case results in a concave-up curve in the region where $R_{0}>1 / K$ with a minimum efficiency equal to $\left(R_{\infty} K-1\right) /\left[K\left(1+R_{\infty}\right)\right]$ as $R_{0} \rightarrow \infty$. The strategy suggested by eqs 8 to 12 to quantify precisely the dynamic kinetic resolution or Curtin-Hammett efficiency for reactions of conformationally related substrates is to determine experimentally the initial product ratio $R_{0}$ in addition to the customary $R_{\infty}$ and $K$ parameters.

Table 5 summarizes the results of the above analyses for minimum estimates of $\epsilon$ using eq 12 on the pyrrolidine systems given in refs 25 and $28 \mathrm{c}$. In all cases, the inequality $R_{0}>R_{\infty}>$ $1 / K$ applies, thus making the Curtin-Hammett efficiency factor lie in the range $\left(R_{\infty} K-1\right) /\left[K\left(1+R_{\infty}\right)\right] \leq \epsilon_{\mathrm{DKR}} \leq 1$. From this analysis, it can be seen that 1-methyl-2-(2'-tert-butylphenyl)pyrrolidine exhibits the best Curtin-Hammett behavior with an efficiency of at least $78 \%$. This is consistent with the observation that reaction takes place preferentially from the conformer having the lone pair of electrons on nitrogen and the $N$-methyl group in anti and syn relationships to the 2-aryl group, respectively. This configuration is less sterically demanding from the point of view of the incoming electrophile. This means that the more thermodynamically stable conformer with the opposite configuration must first change its orientation to the above reactive form before $N$-methylation occurs.

The above analysis can also be used to determine lower-limit estimates of $\epsilon$ for the benzylation of $N$-alkyl piperidines ${ }^{29}$ and 
TABLE 5: Reevaluation of Seeman's $N$-Methylation Reactions of 1-Methyl-2-arylpyrrolidines with Methyliodide Obeying Scheme $1^{a}$

\begin{tabular}{|c|c|c|c|}
\hline 1-methyl-2-arylpyrrolidines & $\begin{array}{l}\text { final product } \\
\text { ratio }^{b}, R_{\infty}\end{array}$ & $\begin{array}{l}\text { equilibrium } \\
\text { constant }^{c}, K\end{array}$ & $\begin{array}{c}\text { minimum } \\
\text { estimates } \\
\text { of } \epsilon_{\mathrm{DKR}}{ }^{d}\end{array}$ \\
\hline 1-methyl-2-phenylpyrrolidine & 0.58 & 17 & 0.33 \\
\hline 1-methyl-2-(2'-methylphenyl)-pyrrolidine & 0.71 & $>30$ & 0.40 \\
\hline 1-methyl-2-(2'-ethylphenyl)-pyrrolidine & 0.77 & $>30$ & 0.42 \\
\hline 1-methyl-2-(2'-isopropylphenyl)-pyrrolidine & 0.77 & $>30$ & 0.42 \\
\hline 1-methyl-2-(2'-t-butylphenyl)-pyrrolidine & 3.57 & $>40$ & 0.78 \\
\hline 1-methyl-2-(2'-pyridyl)pyrrolidine & 0.50 & 15 & 0.29 \\
\hline 1-methyl-2-(3'-pyridyl)pyrrolidine & 0.67 & 15 & 0.36 \\
\hline 1-methyl-2-(4'-pyridyl)pyrrolidine & 0.91 & 15 & 0.44 \\
\hline
\end{tabular}

${ }^{a}$ Data taken from refs 25 and $28 .{ }^{b}$ Defined as the reciprocal of values given in refs 25 and 28 to conform to Scheme 1 defined in this work. ${ }^{c}$ Defined as the ratio of the anti conformer to the syn conformer with respect to the $N$-methyl and 2-aryl groups. ${ }^{d}$ Calculated using $\epsilon_{\min }=\left(R_{\infty} K\right.$ $-1) /\left[\left(1+R_{\infty}\right) K\right]$

TABLE 6: Reevaluation of Katritzky's Benzylation Reactions of 1-Alkyl-4-phenylpiperidines with para-Substituted Benzyl Chlorides Obeying Scheme $\mathbf{1}^{a}$

\begin{tabular}{|c|c|c|c|c|c|c|c|}
\hline \multirow[b]{2}{*}{ alkyl group } & \multirow[b]{2}{*}{$\begin{array}{c}\text { para } \\
\text { substituent }\end{array}$} & \multicolumn{3}{|c|}{ acetonitrile solvent } & \multicolumn{3}{|c|}{ acetone solvent } \\
\hline & & $\begin{array}{l}\text { final product } \\
\text { ratio }^{b}, R_{\infty}\end{array}$ & $\begin{array}{l}\text { equilibrium } \\
\text { constant }^{c}, K\end{array}$ & $\begin{array}{c}\text { minimum } \\
\text { estimates } \\
\text { of } \epsilon_{\mathrm{DKR}}{ }^{d}\end{array}$ & $\begin{array}{l}\text { final product } \\
\text { ratio }^{b}, R_{\infty}\end{array}$ & $\begin{array}{l}\text { equilibrium } \\
\text { constant }^{c}, K\end{array}$ & $\begin{array}{c}\text { minimum } \\
\text { estimates } \\
\text { of } \epsilon_{\mathrm{DKR}}{ }^{d}\end{array}$ \\
\hline \multirow[t]{2}{*}{$\mathrm{Me}$} & $\mathrm{MeO}$ & 1.1 & 3.0 & 0.36 & 3.0 & 3.1 & 0.67 \\
\hline & $\mathrm{H}$ & 2.0 & 3.1 & 0.56 & 6.3 & 3.0 & 0.82 \\
\hline \multirow{4}{*}{$\mathrm{Et}$} & $\mathrm{NO}_{2}$ & 2.4 & 3.0 & 0.61 & 7.8 & 3.1 & 0.85 \\
\hline & $\mathrm{MeO}$ & 1.3 & 7.9 & 0.51 & 6.3 & 8.4 & 0.85 \\
\hline & $\mathrm{H}$ & 1.9 & 8.2 & 0.61 & 13.8 & 8.2 & 0.92 \\
\hline & $\mathrm{NO}_{2}$ & 3.4 & 8.1 & 0.74 & 19.4 & 8.2 & 0.94 \\
\hline \multirow[t]{2}{*}{$i \operatorname{Pr}$} & $\mathrm{MeO}$ & 1.5 & 19.2 & 0.58 & 16.5 & 19.1 & 0.94 \\
\hline & $\mathrm{H}$ & 2.2 & 19.1 & 0.67 & 36.1 & 18.7 & 0.97 \\
\hline
\end{tabular}

${ }^{a}$ Data taken from ref 29. ${ }^{b}$ Defined as the ratio of the equatorial benzylated product to the axial benzylated product. ${ }^{c}$ Defined as the ratio of the equatorial to axial piperidine conformers with respect to the $N$-alkyl group. ${ }^{d}$ Calculated using $\epsilon_{\min }=\left(R_{\infty} K-1\right) /\left[\left(1+R_{\infty}\right) K\right]$.

TABLE 7: Reevaluation of Moreno-Mañas' Hydride Reduction Reactions of Sterically Hindered 1,3-Diketones with Lithium Aluminum Hydride Obeying Scheme $1^{a}$

\begin{tabular}{|c|c|c|c|c|c|c|}
\hline \multicolumn{3}{|c|}{ substituent pattern ${ }^{b}$} & \multirow{2}{*}{$\begin{array}{l}\text { final product } \\
\text { ratio }^{c}, R_{\infty}\end{array}$} & \multirow{2}{*}{$\begin{array}{l}\text { equilibrium } \\
\text { constant }^{d}, K\end{array}$} & \multirow[b]{2}{*}{$R_{\infty} K$} & \multirow{2}{*}{$\begin{array}{c}\text { minimum } \\
\text { estimates } \\
\text { of } \epsilon_{\mathrm{DKR}}{ }^{e}\end{array}$} \\
\hline $\mathrm{R}_{1}$ & $\mathrm{R}_{2}$ & $\mathrm{R}_{3}$ & & & & \\
\hline $\mathrm{Me}$ & $\mathrm{H}$ & 1-Ada & 0.33 & 2.8 & 0.95 & 0.038 \\
\hline $\mathrm{Me}$ & $\mathrm{Me}$ & 1-Ada & 0.053 & 3.3 & 0.17 & 0.79 \\
\hline $\mathrm{Me}$ & $\mathrm{H}$ & $t-\mathrm{Bu}$ & 0.35 & 2.8 & 1.0 & $0^{f}$ \\
\hline $\mathrm{Me}$ & $\mathrm{H}$ & $c-\mathrm{C}_{6} \mathrm{H}_{11}$ & 0.31 & 7.6 & 2.33 & 0.13 \\
\hline$t-\mathrm{Bu}$ & $\mathrm{H}$ & 1-Ada & 1.56 & 0.85 & 1.33 & 0.15 \\
\hline
\end{tabular}

${ }^{a}$ Data taken from ref 26. ${ }^{b}$ Structures of 1,3-diketones given in ref 26. ${ }^{c}$ Defined as the ratio of diol product originating from the high dipole moment diketo conformer to the diol product originating from the low dipole moment diketo conformer. ${ }^{c}$ Defined as the ratio of the low dipole moment diketo conformer to the high dipole moment diketo conformer. ${ }^{e}$ Calculated using $\epsilon_{\min }=\left(1-R_{\infty} K\right) /\left(1+R_{\infty}\right)$ for entries 1 and 2 , and using $\epsilon_{\min }=\left(R_{\infty} K-1\right) /\left[\left(1+R_{\infty}\right) K\right]$ for entries 4 and $5 .{ }^{f}$ Calculated directly from eq 12 since $R_{\infty} K=1$.

the hydride reduction of sterically hindered 1,3-diketones. ${ }^{26}$ Tables 6 and 7 summarize the results of these analyses. The higher Curtin-Hammett efficiency observed in acetone over acetonitrile for the benzylation reaction supports the conclusion of predominant equatorial attack in this solvent. In the case of the hydride reduction reaction, estimated minimum efficiencies near zero indicate anti-Curtin-Hammett behavior and are consistent with product ratios paralleling substrate ratios at equilibrium for those 1,3-diketones that are sterically congested.

In summary, the best way to obtain a priori experimental values of $\epsilon$ for chemical systems obeying Scheme 1 where substrates are interconverting conformers is to determine $K, R_{\infty}$, and $R_{0}$ directly. This involves carrying out experiments using time-resolved techniques that allow both the total and individual concentration-time profiles of products to be monitored from initial equilibrium mixtures of substrates. Estimates of these parameters can then be used directly in eq 12 to obtain $\epsilon$. This strategy can also be used as a check on the graphical analysis based on eqs 3 and 5 for chemical systems obeying Scheme 1, where substrate ratios can be easily manipulated such as in the case of stereoisomerically related substrates. In this case, $\epsilon$ is obtained from the key rate constant ratios $r, u$, and $v$ using eq 7.

\section{Conclusions}

The analysis presented in this work has achieved a unifying description of dynamic kinetic resolution. Several important kinetic schemes have been solved analytically, and their product concentration-time profiles have been determined. New linear relationships are presented that allow for the first time an experimental determination of relative energy barriers under any set of reaction conditions beyond the Curtin-Hammett limit by means of product studies. A new efficiency parameter has been introduced that allows the dynamic kinetic resolution and Curtin-Hammett efficiency to be quantified precisely. Graphical simulations have been used to suggest strategies for optimizing dynamic kinetic resolution in the syntheses of important stereochemical products. The experimental determination of $R_{0}$ in addition to $K$ and $R_{\infty}$ is shown to be a necessary parameter for determining Curtin-Hammett efficiencies for reactions of equilibrium mixtures of conformationally related substrates. Practicing organic chemists need to concern themselves with the linear relationships in eqs 3 and 5, the results given in Tables 2 and S3 for the initial and final product ratios, the graphical treatments described to quantify and optimize dynamic kinetic resolution, and eqs 7 and 12, which quantify the efficiency parameter $\epsilon$. 
Acknowledgment. I thank Mr. Subakar Paramanantham for a preliminary verification of the kinetic analysis for Scheme 1. Parts of this work were presented at the $85^{\text {th }}$ Canadian Society of Chemistry Conference and Exhibition, University of British Columbia, Vancouver, BC, June 1-6, 2002.

Supporting Information Available: Relevant inverse Laplace transforms used in solving simple and complex kinetic schemes under first-order and pseudo first-order conditions; complete solutions for the time dependencies of products in Schemes 1 to 13 using the Laplace transform method; the limits of product concentration-time profiles and their derivatives for Scheme 1 at initial and final times; initial and final product ratios and excesses for variants of Scheme 1; and final product ratios and excesses for variants of Scheme 1 under Curtin-Hammett and anti-Curtin-Hammett conditions. This material is available free of charge via the Internet at http://pubs.acs.org.

\section{Appendix}

Derivation of Winstein-Holness Equation from Equations 1c and $\mathbf{d}$ for Scheme 1. From eqs $1 \mathrm{c}$ and d, the time profile for the total product concentration, $[\mathrm{P}]$, is given by

$$
[\mathrm{P}]=[\mathrm{PX}]+[\mathrm{PY}]=C_{1} \mathrm{e}^{-\gamma_{1} t}+C_{2} \mathrm{e}^{-\gamma_{2} t}+C_{3}
$$

where

$$
\begin{gathered}
C_{1}=\frac{\left(a k_{3}+b k_{4}-(a+b) \gamma_{2}\right)}{\left(\gamma_{2}-\gamma_{1}\right)} \\
C_{2}=\frac{\left(-a k_{3}-b k_{4}+(a+b) \gamma_{1}\right)}{\left(\gamma_{2}-\gamma_{1}\right)}
\end{gathered}
$$

and

$$
C_{3}=a+b
$$

Similarly, from eqs $1 \mathrm{a}$ and $\mathrm{b}$, the time profile for the total substrate concentration, $[\mathrm{S}]$, is given by

$$
[\mathrm{S}]=[\mathrm{X}]+[\mathrm{Y}]=D_{1} \mathrm{e}^{-\gamma_{1} t}+D_{2} \mathrm{e}^{-\gamma_{2} t}
$$

where

$$
D_{1}=\frac{\left(a k_{4}+b k_{3}+(a+b)\left(k_{1}+k_{2}-\gamma_{1}\right)\right)}{\left(\gamma_{2}-\gamma_{1}\right)}
$$

and

$$
D_{2}=-\frac{\left(a k_{4}+b k_{3}+(a+b)\left(k_{1}+k_{2}-\gamma_{2}\right)\right)}{\left(\gamma_{2}-\gamma_{1}\right)}
$$

In the general case, double-exponential behavior is expected when monitoring the appearance of total product or the disappearance of total substrate. In the fortuitous case when $\gamma 1$ $=\gamma 2=\gamma$, preexponential factors in eqs A1 and A2 can be summed to yield the corresponding simple first-order expressions

$$
[\mathrm{P}]=(a+b)\left(1-\mathrm{e}^{-\gamma t}\right)
$$

and

$$
[\mathrm{S}]=(a+b) \mathrm{e}^{-\gamma t}
$$

Such relationships have been found to be adequate in describing kinetic data for chemical systems discussed in refs 25-29. Comparing the general forms of $C_{1}$ with $C_{2}$ shows that $a k_{3}+$ $b k_{4}=C_{1}\left(\gamma_{2}-\gamma_{1}\right)+(a+b) \gamma_{2}=(a+b) \gamma_{1}-C_{2}\left(\gamma_{2}-\gamma_{1}\right)$. When $\gamma 1=\gamma 2=\gamma$, this reduces to $a k_{3}+b k_{4}=(a+b) \gamma$. If the substrates are initially at equilibrium, then $K=b / a$ and

$$
\gamma=\frac{\left(a k_{3}+b k_{4}\right)}{(a+b)}=\frac{\left(k_{3}+K k_{4}\right)}{(1+K)}
$$

which is the Winstein-Holness equation. ${ }^{17}$

\section{References and Notes}

(1) (a) Stephens, T. Chem. Br. 2001, 37 (11), 38. (b) Muller, G. W CHEMTECH 1997, 27, 21. (c) Fabro, S.; Smith, R. L.; Williams, R. T. Nature (London) 1967, 215, 296.

(2) (a) Faber, K.; Kroutil, W. Tetrahedron: Asymmetry 2002, 13, 377. (b) Keith, J. M.; Larrow, J. F.; Jacobsen, E. N. Adv. Synth. Catal. 2001, 343, 5. (c) Cook, G. R. Curr. Org. Chem. 2000, 4, 869. (d) Eames, J. Angew. Chem., Int. Ed. 2000, 39, 885. (e) Strauss, U. T.; Felfer, U.; Faber, K. Tetrahedron: Asymmetry 1999, 10, 107. (f) Hoveyda, A. H.; Didink, M. T. Curr. Org. Chem. 1998, 2, 489. (g) Blackmond, D. G. CATTECH 1998, 2 17

(3) (a) Huerta, F. F.; Minidis, A. B. E.; Bäckvall, J. E. Chem. Soc. Rev. 2001, 30, 321. (b) Faber, K. Chem.-Eur. J. 2001, 7, 5005. (c) Pesti, J. A.; Yin, J.; Lin-hua, Z.; Anzalone, L. J. Am. Chem. Soc. 2001, 123, 11075. (d) Ben, R. N.; Durst, T. J. Org. Chem. 1999, 64, 7700. (e) Ward, R. S. Selectivity in Organic Synthesis; Wiley: New York, 1999; Chapter 6. (f) El Gihani, M. T.; Williams, J. M. Curr. Opin. Chem. Biol. 1999, 3, 11. (g) Parker, R. J.; Williams, J. M. J. Recent Res. Dev. Org. Bioorg. Chem. 1999, 3, 47. (h) O'Meara, J. A.; Gardee, N.; Jung, M.; Ben, R. N.; Durst, T. J. Org. Chem. 1998, 63, 3117. (i) Stecher, H.; Faber, K. Synthesis 1997, 1. (j) Sturmer, R. Angew. Chem., Int. Ed. Engl. 1997, 36, 1173. (k) Caddick, S.; Jenkins, K. Chem. Soc. Rev. 1996, 25, 447. (1) Ward, R. S. Tetrahedron: Asymmetry 1995, 6, 1475. (m) Kagan, H. B.; Fiaud, J. C. In Topics in Stereochemistry; Eliel, E. L., Wilen, S. H., Eds.; Wiley \& Sons: New York, 1988; Vol. 18, pp 249-330. (n) O’Meara, J. A.; Jung, M.; Durst, T. Tetrahedron Lett. 1995, 36, 5096. (o) O’Meara, J. A.; Jung, M.; Durst, T. Tetrahedron Lett. 1995, 36, 2559.

(4) Beak, P.; Anderson, D. R.; Curtis, M. D.; Laumer, J. M.; Pippel, D. J.; Weisenburger, G. A. Acc. Chem. Res. 2000, 33, 715.

(5) (a) Curtin, D. Y. Rec. Chem. Prog. 1954, 15, 111. There is evidence that ideas put forward by Curtin and Hammett pertaining to Scheme 1 were in fact published earlier in 1907 by Solomon Acree (Acree, S. F. Am. Chem. J. 1907, 38, 1). Acree studied the oxygen and nitrogen alkylations of tautomeric mixtures of 1-phenyl urazoles with diazomethane in ether solution. He correctly concluded that "such reactions do not give us decisive evidence in regard to the relative amounts of the enol and keto forms in any given amide group in which the change from one tautomeric form to the other is very rapid in comparison with the reactions between the two forms and the alkylating reagents." Furthermore, he derived an expression, identical in form to the Winstein-Holness equation, for the observed secondorder rate constant for the appearance of total product as a function of the relative populations of the urazole enol and keto forms. The work by Acree was cited neither in the works of Curtin, Hammett, or Winstein on this subject nor in the review by Seeman (see ref 7b). (b) Eliel, E. L.; Wilen, S. H.; Mander, L. N. Stereochemistry of Organic Compounds; Wiley: New York, 1994; pp 647-655. (c) Carroll, F. A. Perspectives in Structure and Mechanism in Organic Chemistry; Brooks/Cole Publishing Co.: New York, 1998; pp 345-349. (d) Carey, F. A.; Sundberg, R. J. Advanced Organic Chemistry, Part A: Structure and Mechanism; Plenum Publishing: New York, 1993; pp 209-216.

(6) (a) Zefirov, N. S. Tetrahedron 1977, 33, 2719. (b) Zefirov, N. S.; Palyulin, V. A. J. Org. Chem. USSR 1979, 15, 980.

(7) (a) Seeman, J. I.; Farone, W. A. J. Org. Chem. 1978, 43, 1854. (b) Seeman, J. I. Chem. Rev. 1983, 83, 83. (c) Perrin, C. L.; Seeman, J. I. J. Org. Chem. 1984, 49, 2887. (d) Seeman, J. I. J. Chem. Educ. 1986, 63, 42.

(8) (a) Matsen, F. A.; Franklin, J. L. J. Am. Chem. Soc. 1950, 72, 3337. (b) McLaughlin, E.; Rozett, R. W. J. Chem. Educ. 1972, 49, 482.

(9) (a) Luukas, T. O.; Girard, C.; Fenwick, D. R.; Kagan, H. B. J. Am Chem. Soc. 1999, 121, 9299. (b) Guillaneaux, D.; Zhao, S.-H.; Samuel, O.; Rainford, D.; Kagan, H. B. J. Am. Chem. Soc. 1994, 116, 9430.

(10) Kitamura, M.; Suga, S.; Oka, H.; Noyori, R. J. Am. Chem. Soc. 1998, 120,9800 .

(11) (a) Blackmond, D. G. J. Am. Chem. Soc. 2001, 123, 545. (b) Blackmond, D. G. Acc. Chem. Res. 2000, 33, 402. (c) Blackmond, D. G.; Rosner, T.; Neugebauer, T.; Reetz, M. T. Angew. Chem., Int. Ed. 1999, 38, 2196. (d) Blackmond, D. G. J. Am. Chem. Soc. 1998, 120, 13349. (e) J. Am. Chem. Soc. 1997, 119, 12939. 
(12) Johnson, D. W., Jr.; Singleton, D. A. J. Am. Chem. Soc. 1999, 121 9307.

(13) (a) Kitamura, M.; Tokunaga, M.; Noyori, R. Tetrahedron 1993 49, 1853. (b) Noyori, R.; Tokunaga, M.; Kitamura, M. Bull. Chem. Soc Jpn. 1995, 68, 36.

(14) (a) Chen, C.-S.; Fujimoto, Y.; Girdaukas, G.; Sih, C. J. J. Am. Chem. Soc. 1982, 104, 7294. (b) Balavoine, G.; Moradpour, A.; Kagan, H. B. J. Am. Chem. Soc. 1974, 96, 5152. (c) Kitamura, M.; Tokunaga, M.; Noyori, R. J. Am. Chem. Soc. 1993, 115, 144. (d) Martin, V. S.; Woodard, S. S.; Katsuki, T.; Yamada, Y.; Ikeda, M.; Sharpless, K. B. J. Am. Chem. Soc. 1981, 103, 6237. (e) Lu, Y.; Zhao, X.; Chen, Z.-N. Tetrahedron: Asymmetry 1995, 6, 1093

(15) (a) Wagner, J. G. Fundamentals of Clinical Pharmacokinetics; Drug Intelligence Publications: Hamilton, IL, 1975. (b) Boroujerdi, M. Phar macokinetics: Principles and Applications; McGraw-Hill: New York, 2002. (c) Andraos, J. J. Chem. Educ. 1999, 76, 1578.

(16) Thomas, G. B., Jr. Calculus and Analytical Geometry; AddisonWesley: Reading, MA, 1972; pp 838-844.

(17) Winstein, S.; Holness, N. J. J. Am. Chem. Soc. 1955, 77, 5562.

(18) Hutchings, R. O. J. Org. Chem. 1977, 42, 920.

(19) Monnier, M.; Aycard, J. P. Can. J. Chem. 1979, 57, 1257.
(20) Jaeger, D. A.; Broadhurst, M. D.; Cram, D. J. J. Am. Chem. Soc 1979, 101, 717.

(21) (a) Guthrie, R. D.; Jaeger, D. A.; Meister, W.; Cram, D. J. J. Am. Chem. Soc. 1971, 93, 5137. (b) Jaeger, D. A.; Cram, D. J. J. Am. Chem. Soc. 1971, 93, 5153.

(22) The analytical solution given in eqs $1 \mathrm{a}-\mathrm{d}$ have been verified using the symbolic logic program Mathematica (Wolfram Research).

(23) (a) Gold, V. Pure Appl. Chem. 1979, 51, 1725. (b) Gold, V. Pure Appl. Chem. 1983, 55, 1281 .

(24) Singleton, D. A.; Hang, C. J. Am. Chem. Soc. 1999, 121, 11885.

(25) Seeman, J. I.; Secor, H. V.; Hartung, H.; Galzerano, R. J. Am. Chem. Soc. 1980, 102, 7741

(26) Moreno-Mañas, M.; Galvez, N.; Lloris, M. E.; Marquet, J.; Siani, A. C. Tetrahedron 1992, 48, 3603 .

(27) Dolbier, W. R.; Enoch, H. O. J. Am. Chem. Soc. 1977, 99 , 4532 .

(28) (a) Whidby, J. F.; Seeman, J. I. J. Org. Chem. 1976, 41, 1585. (b) Seeman, J. I.; Secor, H. V.; Bassfield, R. L.; Whidby, J. F. Tetrahedron Lett. 1978, 1901. (c) Seeman, J. I. Pure Appl. Chem. 1987, 59, 1661

(29) Duke, R. P.; Jones, R. A. Y.; Katritzky, A. R. J. Chem. Soc., Perkin Trans. 2 1973, 1553 